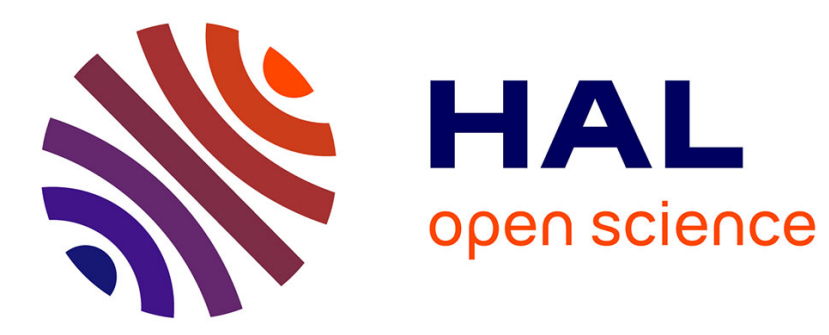

\title{
An improved time domain linear sampling method for Robin and Neumann obstacles
}

\author{
Houssem Haddar, Armin Lechleiter, Simon Marmorat
}

\section{To cite this version:}

Houssem Haddar, Armin Lechleiter, Simon Marmorat. An improved time domain linear sampling method for Robin and Neumann obstacles. Applicable Analysis, 2013, pp.1-22. 10.1080/00036811.2013.772583 . hal-00768725

\section{HAL Id: hal-00768725}

\section{https://hal.inria.fr/hal-00768725}

Submitted on 15 Jan 2013

HAL is a multi-disciplinary open access archive for the deposit and dissemination of scientific research documents, whether they are published or not. The documents may come from teaching and research institutions in France or abroad, or from public or private research centers.
L'archive ouverte pluridisciplinaire $\mathbf{H A L}$, est destinée au dépôt et à la diffusion de documents scientifiques de niveau recherche, publiés ou non, émanant des établissements d'enseignement et de recherche français ou étrangers, des laboratoires publics ou privés. 


\title{
An improved time domain linear sampling method for Robin and Neumann obstacles
}

\author{
Houssem Haddar* $\quad$ Armin Lechleiter ${ }^{\dagger} \quad$ Simon Marmorat ${ }^{\ddagger}$
}

October 17, 2012

\begin{abstract}
We consider inverse obstacle scattering problems for the wave equation with Robin or Neumann boundary conditions. The problem of reconstructing the geometry of such obstacles from measurements of scattered waves in the time domain is tackled using a time domain linear sampling method. This imaging technique yields a picture of the scatterer by solving a linear operator equation involving the measured data for many right-hand sides given by singular solutions to the wave equation. We analyze this algorithm for causal and smooth impulse shapes, we discuss the effect of different choices of the singular solutions used in the algorithm, and finally we propose a fast FFT-based implementation.
\end{abstract}

\section{Introduction}

In this paper we propose and analyze a time domain linear sampling method as an algorithm to solve the inverse scattering problem of reconstructing an obstacle with Robin or Neumann boundary condition from time-dependent near-field measurements of scattered waves. Our algorithm is an improvement of the one introduced in [7] to solve a similar inverse scattering problem for obstacles with Dirichlet boundary conditions. This algorithm is a direct imaging technique that is able to provide geometric information on the obstacle from wave measurements. Crucial ingredients of the method are the near-field operator, a linear integral operator that takes the measured data as integral (and convolution) kernel, and special test functions that are constructed via singular solutions to the wave equation. Using these ingredients, the method checks whether a point belongs to the obstacle by checking whether these test functions belong to the range of the measurement operator. Plotting the reciprocal of the norm of the corresponding pre-image yields a picture of the scattering object.

In addition to the analysis of a different scattering problem, we provide in the present work a substantial improvement of the method originally introduced in [7] on both theoretical and numerical levels. More specifically, we shall analyze the method for incident waves generated by pulses with bounded spectrum. Moreover, adapting the function space setting to this type of data allows us to provide a simpler analysis. On the numerical side, we shall present a fast implementation of the inversion algorithm that relies on a FFT-based evaluation of the near-field operator. We

${ }^{*}$ DEFI, INRIA Saclay-Ile-de-France and Ecole Polytechnique, 91128 Palaiseau, France

${ }^{\dagger}$ Center for Industrial Mathematics, University of Bremen, 28359 Bremen, Germany

${ }^{\ddagger}$ POEMS, INRIA Rocquencourt, 78153 Le Chesnay, France 
also show, by mixing the use of monopoles and dipoles as test functions, the possibility of simultaneously reconstructing Dirichlet, Neumann and Robin obstacles (see 22] for somewhat related considerations in the frequency domain).

The time domain linear sampling method that we treat in this paper has a fixed-frequency domain counterpart that has been intensively studied in the last years, see, e.g., 6] for an introduction. Recently, many different ways of setting up inverse scattering algorithms that are able to cope with measurements extending the traditional fixed-frequency assumption appeared in the literature. Those include multi-frequency versions of the linear sampling method [14], or generally speaking multi-frequency versions of sampling methods [19, 1, 5, 13. Further recent work on inverse scattering in the time domain includes algorithms inspired by time reversal and boundary control techniques [4, 3], partly extending to inverse problems with unknown background sound speed [21].

Three points about the relevance of time domain linear sampling algorithm seem worth to be discussed in advance: First, in contrast to usual multi-frequency approaches, the method avoids the need to synthesize several multi-frequency reconstructions, since one reconstruction is directly computed from the time domain data. Second, the method is in principle able to reconstruct the shape of all connected components of the obstacle (assuming that the complement of the obstacle is connected). Of course, this ability decreases with increasing noise level and a decreasing number of emitters and receivers. The price to pay for the ability to reconstruct more than the convex hull of the obstacle is that the time measurements of the scattered fields must in principle be unlimited. In practice we stop the measurements when significantly much wave energy has left the computational domain. Third, a possible alternative to the proposed method would be to take a single Fourier transform in time of the data, and then to use an inversion algorithm at fixed-frequency. This way of handling the data might yield faster inversion algorithms, however, the optimal choice of the transformation frequency depends on the unknown obstacle and might in general not be obvious to guess.

The structure of this paper is as follows. In Section 2 we first present the inverse problem and formally outline the inversion method. We then analyze the forward time domain scattering problem using a Laplace-Fourier transformation in time. The last part of this section is dedicated to some auxiliary results needed in the analysis of the inversion algorithm. Section 3 contains the main theoretical result that motivated the time domain linear sampling method. The numerical implementation and validation of the algorithm is presented in Section 4. Finally, an appendix is added with some results on retarded potentials that would facilitate the reading of the technical parts of the proofs.

\section{Presentation of the forward and inverse scattering problem}

\subsection{Formal presentation of the inversion method}

The inverse problem we consider is to reconstruct an obstacle $\Omega_{-} \subset \mathbb{R}^{3}$, assumed to be bounded and Lipschitz with connected complement, from time domain near-field measurements of scattered waves on some measurement surface $\Gamma_{m} \subset \mathbb{R}^{3} \backslash \overline{\Omega_{-}}$. Let us denote by $u(t, x ; y)$ the scattered field for an incident point source $u^{i}(\cdot, \cdot ; y)$ emitted at $y \in \Gamma_{i}$, another surface in $\mathbb{R}^{3} \backslash \overline{\Omega_{-}}$, possibly equal to $\Gamma_{m}$. This incident field is convolution in time of the fundamental solution of the wave equation with a pulse $\chi$,

$$
u^{i}(t, x ; y):=\frac{\chi(t-|x-y|)}{4 \pi|x-y|}, \quad t \in \mathbb{R}, x \in \mathbb{R}^{3} \backslash\{y\}
$$


Let $\Omega_{+}:=\mathbb{R}^{3} \backslash \bar{\Omega}_{-}$and denote by $n$ the normal vector defined on $\Gamma:=\partial \Omega_{-}$directed to $\Omega_{+}$. The scattered field $u(\cdot, \cdot ; y)$ solves the following scattering problem with a Robin boundary condition on the boundary of the scatterer,

$$
\begin{cases}\partial_{t t} u(t, x)-\Delta u(t, x)=0, & t \in \mathbb{R}, x \in \Omega_{+}, \\ \partial_{n} u(t, x)-\alpha \partial_{t} u(t, x)=-\left(\partial_{n} u^{i}(t, x ; y)-\alpha \partial_{t} u^{i}(t, x ; y)\right), & t \in \mathbb{R}, x \in \partial \Omega_{+},\end{cases}
$$

subject to a causality condition: $u(t, x)=0$ for $t<0$ and $x \in \Omega_{+}$. The impedance $\alpha$ is a positive bounded function on the boundary of the scatterer that we choose to be merely space-dependent, for simplicity. (More complicated frequency-dependent impedance models could be treated.) The task is hence to reconstruct $\Omega_{-}$from the partial knowledge of the scattered waves

$$
\left\{u(t, x ; y): t \in \mathbb{R}, x \in \Gamma_{m}, y \in \Gamma_{i}\right\}
$$

compare Figure 1. We exploit the linearity of the direct scattering problem by introducing the

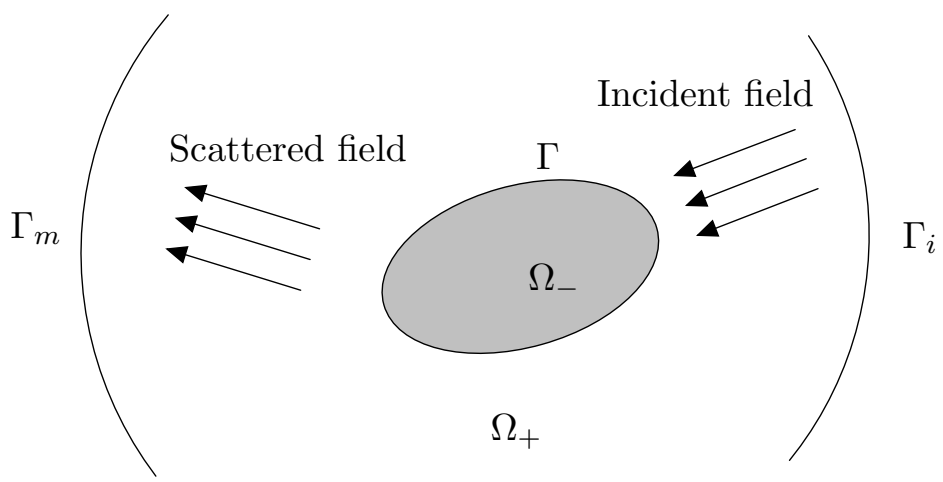

Figure 1: Geometry of the wave scattering problem: the obstacle $\Omega_{-}$with boundary $\Gamma$ scatters incident waves emitted from $\Gamma_{i}$. The scattered fields are recorded on $\Gamma_{m}$.

near-field operator, a linear time convolution with kernel $u(t, x ; y)$,

$$
(N \psi)(t, x)=\int_{\mathbb{R}} \int_{\Gamma_{i}} u(t-\tau, x ; y) \psi(\tau, y) d y d \tau, \quad(t, x) \in \mathbb{R} \times \Gamma_{m} .
$$

The linear sampling algorithm we propose, roughly speaking, checks whether point sources (monopoles $u^{i}(t, x ; y)$ or corresponding dipoles as later introduced in Section 3 belong to the range of $N$ by approximately solving a near-field equation, e.g., $N g=\left.u^{i}(\cdot, \cdot ; z)\right|_{\Gamma_{m} \times \mathbb{R}}$. This test would provide an image of the scatterer by plotting the norm of $g=g_{z}$ in dependence of the source point $z$. In order to theoretically justify this approach one therefore has to first indicate the domain of definition of the operator $N$ and then analyze the range of this operator. These steps indeed rely on the analysis of the forward scattering problem described in (1). 


\subsection{Analysis of the forward scattering problem}

The scattering problem (1) is a special case of the following problem

$$
\begin{cases}\partial_{t t} u(t, x)-\Delta u(t, x)=0, & t \in \mathbb{R}, x \in \Omega_{+} \\ \partial_{n} u(t, x)-\alpha \partial_{t} u(t, x)=g(t, x), & t \in \mathbb{R}, x \in \Gamma\end{cases}
$$

where $g$ is a given (causal) boundary data. We shall analyze this problem using a Fourier-Laplace method as in [2, 15, 11] (see also [23, Section 39], [10, Chapter 16]). For a Banach space $X$ we denote by $\mathcal{D}^{\prime}(\mathbb{R}, X)$ and by $\mathcal{S}^{\prime}(\mathbb{R}, X)$ the space of $X$-valued distributions and tempered distributions on the real line, respectively. For $\sigma \in \mathbb{R}$ we set

$$
\mathcal{L}_{\sigma}^{\prime}(\mathbb{R}, X)=\left\{f \in \mathcal{D}^{\prime}(\mathbb{R}, X) \text { such that } e^{-\sigma t} f \in \mathcal{S}^{\prime}(\mathbb{R}, X)\right\} .
$$

For $f \in \mathcal{L}_{\sigma}^{\prime}(\mathbb{R}, X)$ such that $e^{-\sigma t} f \in \mathcal{L}^{1}(\mathbb{R}, X)$, we define the Laplace transform by

$$
\widehat{f}(\omega)=\int_{-\infty}^{\infty} e^{i \omega t} f(t) d t \in X, \omega \in \mathbb{R}+i \sigma .
$$

For $\sigma \in \mathbb{R}$ we denote

$$
\mathbb{C}_{\sigma}:=\{\omega \in \mathbb{C} ; \operatorname{Im}(\omega) \geq \sigma\}
$$

Formally applying the Laplace transform to (2), one observes that $\widehat{u}(\omega, \cdot)$ is a solution of the Helmholtz-like problem

$$
\begin{cases}\left(\Delta+\omega^{2}\right) \widehat{u}(\omega, x)=0, & x \in \Omega_{+}, \\ \partial_{n} \widehat{u}(\omega, x)+i \omega \alpha \widehat{u}(\omega, x)=\widehat{g}(\omega, x), & x \in \Gamma .\end{cases}
$$

In order to analyze problem (2) we shall study first (3) and derive explicit bounds of the solution in terms of $\omega \in \mathbb{C}_{\sigma_{0}}$ for some $\sigma_{0}>0$. Following [15, for a domain $\Omega$ we use the following frequency dependent norm on $H^{1}(\Omega)$ :

$$
\|u\|_{1, \omega, \Omega}:=\sqrt{\int_{\Omega}\left(|\nabla \widehat{u}(\omega, x)|^{2}+|\omega \widehat{u}(\omega, x)|^{2}\right) d x}
$$

which is equivalent to the usual norm of $H^{1}(\Omega)$ if $\omega \neq 0$. Similar frequency dependent norms exist for the trace spaces $H^{ \pm 1 / 2}(\Gamma)$ (see [20] for a general definition) on the boundary $\Gamma=\partial \Omega$. Omitting details, these norms can for instance be defined using the usual spatial Fourier transform $\mathcal{F}$ defined in $\mathcal{S}^{\prime}\left(\mathbb{R}^{2}\right)$, local charts $\Phi_{j}: \mathbb{R}^{2} \rightarrow \Gamma$ and an associated partition of unity $\chi_{j}: \Gamma \rightarrow \mathbb{R}, j=1, \ldots, N$, on $\Gamma$ by

$$
\|\phi\|_{s, \omega, \Gamma}^{2}:=\sum_{j=1}^{N} \int_{\mathbb{R}^{2}}\left(|\omega|^{2}+|\xi|^{2}\right)^{s}\left|\mathcal{F}\left[\left(\chi_{j} \phi\right) \circ \Phi_{j}(\xi)\right]\right|^{2} d \xi, \quad|s|<1, \omega \in \mathbb{R}+i \sigma, \sigma>0 .
$$

The space $H^{s}(\Gamma)$ is then defined for $|s|<1$ as the completion of $C^{\infty}(\Gamma)$ in the norm $\|\cdot\|_{s, \omega, \Gamma}$. When equipped with these norms, the spaces $H^{ \pm 1 / 2}(\Gamma)$ are (as usual) dual to each other for the duality product extending the $L^{2}$ inner product $\langle f, g\rangle_{\Gamma}=\int_{\Gamma} f \bar{g} d s$.

The advantage of these frequency-depending norms is that the constants in the corresponding trace theorem can be bounded independently of $\omega \in \mathbb{C}_{\sigma_{0}}$. To state this result, e.g., in the version from [15, we denote by $\operatorname{tr}_{\Gamma}: H^{1}(\Omega) \rightarrow H^{1 / 2}(\Gamma)$ the trace operator on $H^{1}(\Omega)$. 
Lemma 1. Let $\sigma_{0}>0$.

(1) There exists a positive constant $C$ depending only on $\Omega$ and $\sigma_{0}$ such that

$$
\left\|\operatorname{tr}_{\Gamma} u\right\|_{1 / 2, \omega, \Gamma} \leq C\|u\|_{1, \omega, \Omega} \quad \text { for all } u \in H^{1}(\Omega) \text { and } \omega \in \mathbb{C}_{\sigma_{0}} .
$$

(2) Conversely, there is a trace lifting operator $\operatorname{ext}_{\Gamma}: H^{1 / 2}(\Gamma) \rightarrow H^{1}(\Omega)$ and a positive constant $C$ depending only on $\Omega$ and $\sigma_{0}$ such that

$$
\left\|\operatorname{ext}_{\Gamma} \phi\right\|_{1, \omega, \Omega} \leq C\|\phi\|_{1 / 2, \omega, \Gamma} \quad \text { for all } \phi \in H^{1 / 2}(\Gamma) \text { and } \omega \in \mathbb{C}_{\sigma_{0}} .
$$

Then we have the following result.

Proposition 2. Let $\sigma_{0}>0, \omega \in \mathbb{C}_{\sigma_{0}}$ and assume that $\widehat{g}(\omega, \cdot) \in H^{-1 / 2}(\Gamma)$. Then, problem (3) has a unique solution $\widehat{u}(\omega, \cdot) \in H^{1}\left(\Omega_{+}\right)$. Moreover, there exists a constant $C$ depending only on $\sigma_{0}$ and $\Omega_{+}$such that

$$
\|\widehat{u}(\omega, \cdot)\|_{1, \omega, \Omega_{+}} \leq C|\omega|\|\widehat{g}(\omega, \cdot)\|_{-1 / 2, \omega, \Gamma} .
$$

Proof. Consider the variational formulation associated with (3): $\widehat{u}$ solves $(3)$ if and only if

$$
A(\widehat{u}, v):=\int_{\Omega_{+}}\left[\nabla \widehat{u} \cdot \overline{\nabla v}-\omega^{2} \widehat{u} \bar{v}\right] d x-i \omega \int_{\Gamma} \alpha \widehat{u} \bar{v} d s=\langle\widehat{g}, v\rangle_{\Gamma} \text { for all } v \in H^{1}\left(\Omega_{+}\right) .
$$

Multiplying (5) by $i \bar{\omega}=: i \eta+\sigma$, taking the real part and choosing $v=\widehat{u}$, one obtains

$$
\operatorname{Re}(i \bar{\omega} A(\widehat{u}, \widehat{u}))=\sigma \int_{\Omega_{+}}\left[|\nabla \widehat{u}|^{2}+\left|\omega^{2} \| \widehat{u}\right|^{2}\right] d x+|\omega|^{2} \int_{\Gamma} \alpha|\widehat{u}|^{2} d s \geq \sigma_{0}\|\widehat{u}\|_{1, \omega, \Omega_{+}}^{2} .
$$

This shows that (3) admits a unique solution. The announced estimate now follows from the inequality $\langle\widehat{g}, \widehat{u}\rangle_{\Gamma} \leq\|\widehat{u}\|_{1 / 2, \omega, \Gamma}\|\widehat{g}\|_{-1 / 2, \omega, \Gamma}$, combined with Lemma 1 .

Remark 3. The bounds in Proposition 2 can be improved if $\widehat{g}(\omega, \cdot) \in L^{2}(\Gamma)$, and if there exists a constant $\alpha_{0}>0$ such that $\alpha(x) \geq \alpha_{0}$ for almost all $x \in \Gamma$. Under these assumptions, one easily deduces from (6) the existence of $C$ depending only on $\alpha$ such that

$$
\|\widehat{u}(\omega, \cdot)\|_{1, \omega, \Omega_{+}} \leq C / \sigma_{0}\|\widehat{g}(\omega, \cdot)\|_{0, \omega, \Gamma} .
$$

For $p \in \mathbb{R}, s \in \mathbb{R}$, and $\sigma \in \mathbb{R}$, we then introduce the weighted Sobolev spaces

$$
\begin{aligned}
& H_{\sigma, \Omega}^{p, 1}:=\left\{u \in \mathcal{L}_{\sigma}^{\prime}\left(\mathbb{R}, H^{1}(\Omega)\right): \int_{-\infty+i \sigma}^{\infty+i \sigma}|\omega|^{2 p}\|\hat{u}\|_{1, \omega, \Omega}^{2} d \omega<\infty\right\}, \\
& H_{\sigma, \Gamma}^{p, s}:=\left\{\phi \in \mathcal{L}_{\sigma}^{\prime}\left(\mathbb{R}, H^{s}(\Gamma)\right): \int_{-\infty+i \sigma}^{\infty+i \sigma}|\omega|^{2 p}\|\hat{\phi}\|_{s, \omega, \Gamma}^{2} d \omega<\infty\right\}, \quad|s|<1 .
\end{aligned}
$$

The norms on these (Hilbert) spaces are, respectively,

$$
\begin{aligned}
\|u\|_{H_{\sigma, \Omega}^{p, 1}} & :=\left(\int_{-\infty+i \sigma}^{\infty+i \sigma}|\omega|^{2 p}\|\hat{u}\|_{1, \omega, \Omega}^{2} d \omega\right)^{1 / 2}, \\
\|\phi\|_{H_{\sigma, \Gamma}^{p, s}} & :=\left(\int_{-\infty+i \sigma}^{\infty+i \sigma}|\omega|^{2 p}\|\hat{\phi}\|_{s, \omega, \Gamma}^{2} d \omega\right)^{1 / 2}, \quad|s|<1 .
\end{aligned}
$$

As a consequence of Proposition (2) and the use of Fourier-Laplace transform one gets the following result. 
Proposition 4. Let $\sigma_{0}>0$ and assume that $g \in H_{\sigma_{0}, \Gamma}^{p+1,-1 / 2}$ for some $p \in \mathbb{R}$. Then, problem (2) has a unique solution $u \in H_{\sigma, \Omega_{+}}^{p, 1}$ with $\sigma \geq \sigma_{0}$. Moreover, there exists a constant depending only on $\sigma_{0}$ and $\Omega_{+}$such that

$$
\|u\|_{H_{\sigma, \Omega_{+}}^{p, 1}} \leq C\|g\|_{H_{\sigma, \Gamma}^{p+1,-1 / 2}}
$$

for all $\sigma \geq \sigma_{0}$.

For later use we denote by $G$ the solution operator to problem (2),

$$
G: H_{\sigma, \Gamma}^{p+1,-1 / 2} \rightarrow H_{\sigma, \Omega_{+}}^{p, 1} \text { defined by } G(g)=u,
$$

where $u \in H_{\sigma, \Omega_{+}}^{p, 1}$ is the unique solution of $(2)$ for $\sigma>0$ and $p \in \mathbb{R}$. Proposition 4 ensures that this operator is well-defined and bounded.

Remark 5. We observe that, according to the Paley-Wiener theory (see [15, Theorem 1], or [10]), the uniform bound in (2) with respect to $\omega \in \mathbb{C}_{\sigma_{0}}$ and the fact that if $\omega \mapsto \widehat{g}(\omega, \cdot)$ is holomorphic in $\mathbb{C}_{\sigma_{0}}$ with values in $H^{-1 / 2}(\Gamma)$ then $\omega \mapsto \widehat{u}(\omega, \cdot)$ is holomorphic in $\mathbb{C}_{\sigma_{0}}$ with values in $L^{2}\left(\Omega_{+}\right)$implies that, if $g$ is causal then the unique solution defined in Proposition 4 is also causal.

As a consequence of the previous proposition (and the previous remark), one observes in particular that if $\chi$ is a $C^{p+2}$-function with compact support then the scattering problem (1) has a unique solution in $H_{\sigma, \Omega_{+}}^{p, 1}, \sigma>0$. Moreover, if $\chi$ vanishes for $t \leq T$ then the solution also vanishes for $t \leq T$. We shall refine in the next section this type of results and set up the function space for the operator $N$.

\subsection{Auxiliary Results for the Analysis of the Sampling Algorithm}

The following results aim at giving an appropriate function space setting for the operator $N$. For instance, by (formal) linearity of the scattering problem with respect to the incident field, one observes that $N \psi$ is nothing but the trace on $\Gamma_{m}$ of the solution to (1) with the incident field $u^{i}$ replaced by the (regularized) single layer potential

$$
\left(L_{\Gamma_{i}}^{\chi} \psi\right)(t, x):=\int_{\mathbb{R}} \int_{\Gamma_{i}} \frac{\chi(t-\tau-|x-y|)}{4 \pi|x-y|} \psi(\tau, y) d \sigma_{y} d \tau, \quad(t, x) \in \mathbb{R} \times\left(\mathbb{R}^{3} \backslash \Gamma_{i}\right) .
$$

In this section we provide a couple of auxiliary results related to this potential. For analytical purposes we assume that either $\Gamma_{i}$ and $\Gamma_{m}$ are closed surfaces or else that both are relatively open subsets of closed analytic surfaces. In the latter case, the spaces $H_{\sigma, \Gamma_{i, m}}^{p, \pm 1 / 2}$ have to be adapted, following, e.g., the section in [20] on Sobolev spaces on the boundary. Since this is a standard procedure, we do not go into details and do not denote the adapted spaces explicitly. Later on in the main result we merely consider the $L^{2}$-spaces $H_{\sigma, \Gamma_{i, m}}^{0,0}$ and then this issue is anyway not relevant anymore.

Concerning the pulse function $\chi: \mathbb{R} \rightarrow \mathbb{R}$ we shall assume that it is a non-trivial and causal $C^{3}$-function such that its Laplace transform is holomorphic in $\mathbb{C}_{0}$ and has a cubic decay rate,

$$
|\widehat{\chi}(\omega)| \leq \frac{C}{|\omega|^{3}} \quad \text { for } \omega \in \mathbb{C}_{0} .
$$

This assumption is not strictly necessary, but allows us to use relatively simple function spaces in the main result of this paper. Slower decay rates essentially would change the time regularity of all 
later results. We also quote that this assumption is satisfied by causal $C^{3}$-functions with compact support.

Our results are based on the analysis of retarded potentials. Some key results from [15, 9, 18, 20] are summarized in the appendix. The next proposition also uses the following identity, that can be easily verified for regular densities $\psi$ with compact support,

$$
\left(L_{\Gamma_{i}}^{\chi} \psi\right)(t, x)=\left(\chi * L_{\Gamma_{i}} \psi\right)(t, x)
$$

where the retarded potential $L_{\Gamma_{i}}$ is defined by (see also the appendix)

$$
\left(L_{\Gamma_{i}} \psi\right)(t, x):=\int_{\Gamma_{i}} \frac{\psi(t-|x-y|, y)}{4 \pi|x-y|} d \sigma_{y},(t, x) \in \mathbb{R} \times\left(\mathbb{R}^{3} \backslash \Gamma\right) .
$$

From identity (13), we also observe that $L_{\Gamma_{i}}^{\chi}$ is a time convolution operator, i.e. for regular densities $\psi$ with compact support,

$$
\widehat{L_{\Gamma_{i}}^{\chi} \psi}(\omega, x)=\left(\widehat{L_{\Gamma_{i}}^{\chi}}(\omega) \widehat{\psi}(\omega, \cdot)\right)(x)
$$

for $x \in \mathbb{R} \backslash \Gamma_{i}$ and $\omega \in \mathbb{C}$, where

$$
\widehat{L_{\Gamma_{i}}^{\chi}}(\omega)=\widehat{\chi}(\omega) \widehat{L_{\Gamma_{i}}}(\omega)
$$

and where $\widehat{L_{\Gamma_{i}}}(\omega)$ is defined by 46 .

Proposition 6. Let $p \in \mathbb{R}$ and $\sigma>0$. Then, the operator

$$
L_{\Gamma_{i}}^{\chi}: H_{\sigma, \Gamma_{i}}^{p, 0} \rightarrow H_{\sigma, \Omega_{+}}^{p+2,1}
$$

is bounded and injective. Moreover, the operator

$$
\operatorname{tr}_{\Gamma} L_{\Gamma_{i}}^{\chi}: H_{\sigma, \Gamma_{i}}^{p, 0} \rightarrow H_{\sigma, \Gamma}^{p+2,1 / 2}
$$

is bounded, injective with dense range.

Proof. Let $\psi \in \mathcal{D}\left(\mathbb{R} \times \Gamma_{i}\right)$. Using (16), bound $(50)$ for $\widehat{L_{\Gamma_{i}}}(\omega)$ and assumption 12 imply the existence of a constant $C$ such that, for all $\omega \in \mathbb{R}+i \sigma$

$$
\left\|\widehat{L_{\Gamma_{i}}^{\chi}}(\omega) \widehat{\psi}(\omega, \cdot)\right\|_{1, \omega, \Omega_{+}} \leq C|\omega|\|\widehat{\psi}(\omega, \cdot)\|_{-1 / 2, \omega, \Gamma}
$$

Boundedness of $L_{\Gamma_{i}}^{\chi}$ from $H_{\sigma, \Gamma_{i}}^{p,-1 / 2}$ into $H_{\sigma, \Omega_{+}}^{p+2,1}$ then follows using the definition of these spaces and a density argument. Since, in addition, $H_{\sigma, \Gamma_{i}}^{p, 0}$ is continuously embedded in $H_{\sigma, \Gamma_{i}}^{p,-1 / 2}$, the first claim is proved. The boundedness of the operator $\operatorname{tr}_{\Gamma} L_{\Gamma_{i}}^{\chi}: H_{\sigma, \Gamma_{i}}^{p, 0} \rightarrow H_{\sigma, \Gamma}^{p+2,1 / 2}$ follows using Lemma 1 .

By a density argument we further observe that identity $(15)$ is still satisfied for $\psi \in H_{\sigma, \Gamma_{i}}^{p, \sigma}$, for a.e. $(\omega, x) \in(\mathbb{R}+i \sigma) \times \Omega_{+}$. Consequently, if $L_{\Gamma_{i}}^{\chi} \psi=0$, then

$$
\widehat{\chi}(\omega) \widehat{L_{\Gamma_{i}}}(\omega) \widehat{\psi}(\omega, \cdot)=0 \text { in } \Omega_{+} \text {for a.e. } \omega \in \mathbb{R}+i \sigma .
$$

Our assumptions imply in particular that the zeros of $\widehat{\chi}(\omega), \omega \in \mathbb{R}+i \sigma$, form an at most countable discrete set without finite accumulation point. Hence $\widehat{L_{\Gamma_{i}}}(\omega) \widehat{\psi}(\omega, \cdot)=0$ in $\Omega_{+}$for a.e. $\omega \in \mathbb{R}+i \sigma$. Since the operator $\widehat{L_{\Gamma_{i}}}(\omega): H^{-1 / 2}\left(\Gamma_{i}\right) \rightarrow H^{1}\left(\Omega_{+}\right)$is injective for all $\omega \in \mathbb{R}+i \sigma$ (see Proposition 17 ), 
we obtain $\widehat{\psi}(\omega, \cdot)$ in $\Gamma_{i}$ for a.e. $\omega \in \mathbb{R}+i \sigma$ which implies that $\psi=0$. The injectivity of $\operatorname{tr}_{\Gamma} L_{\Gamma_{i}}^{\chi}$ can be proved in a similar way using the injectivity of the operator $\operatorname{tr}_{\Gamma} \widehat{L_{\Gamma_{i}}}(\omega): H^{-1 / 2}\left(\Gamma_{i}\right) \rightarrow H^{1 / 2}(\Gamma)$ for a.e. $\omega \in \mathbb{R}+i \sigma$ (see Proposition 17 ).

Finally, the denseness of the range of $\operatorname{tr}_{\Gamma} L_{\Gamma_{i}}^{\chi}$ can be seen by showing that the $L^{2}$-adjoint of $\operatorname{tr}_{\Gamma} L_{\Gamma_{i}}^{\chi}$ is injective. Denoting $A^{*}$ the adjoint of an operator $A$, one gets

$$
\left(\operatorname{tr}_{\Gamma} L_{\Gamma_{i}}^{\chi}\right)^{*}=\left(\operatorname{tr}_{\Gamma} L_{\Gamma_{i}}\right)^{*}\left(\chi^{*} *\right):=B
$$

where $\chi^{*}(t)=\chi(-t)$ and where

$$
\left[\left(\operatorname{tr}_{\Gamma} L_{\Gamma_{i}}\right)^{*} v\right](t, y)=\int_{\Gamma} \frac{v(t+|x-y|, y)}{4 \pi|x-y|} d x, \quad(t, y) \in \mathbb{R} \times \Gamma_{i} .
$$

The injectivity of $\left(\operatorname{tr}_{\Gamma} L_{\Gamma_{i}}^{\chi}\right)^{*}$ on $\left(H_{\sigma, \Gamma}^{p+2,1 / 2}\right)^{\prime}=H_{-\sigma, \Gamma}^{-p-2,-1 / 2}$ can be also checked by analyzing the injectivity of the Fourier-Laplace symbol of $\left(\operatorname{tr}_{\Gamma} L_{\Gamma_{i}}^{\chi}\right)^{*}$ on the line $\mathbb{R}-i \sigma$. Defining $\widehat{B}$ as in 15 we simply get

$$
\widehat{B}(\omega)=\widehat{\chi}(-\omega) \operatorname{tr}_{\Gamma_{i}} \widehat{L_{\Gamma}}(-\omega) .
$$

Therefore, following the same lines as above for the injectivity of $\operatorname{tr}_{\Gamma} L_{\Gamma_{i}}^{\chi}$, one obtains the desired result as a consequence of the injectivity of $\operatorname{tr}_{\Gamma_{i}} \widehat{L_{\Gamma}}(-\omega): H^{-1 / 2}\left(\Gamma_{i}\right) \rightarrow H^{1 / 2}(\Gamma)$ for a.e. $\omega \in \mathbb{R}-i \sigma$ (see Proposition 17).

We need to derive estimates for the interior and exterior Dirichlet problem in the spaces $H_{\sigma, \Omega_{ \pm}}^{p, s}$. Let $\omega \in \mathbb{R}+i \sigma, \sigma>0$, and consider $f \in H^{1 / 2}(\Gamma)$, a boundary datum for the following problem: Find $v \in H^{1}\left(\Omega_{ \pm}\right)$such that

$$
\begin{cases}\left(\Delta+\omega^{2}\right) v(x)=0, & x \in \Omega_{ \pm} \\ v(x)=f(x), & x \in \Gamma\end{cases}
$$

Lemma 7. Problem (18) admits a unique solution $v \in H^{1}\left(\Omega_{ \pm}\right)$. Moreover, there exists a constant $C>0$ depending only on $\sigma>0$ and $\Gamma$ such that

$$
\|v\|_{1, \omega, \Omega_{ \pm}} \leq C|\omega|\|f\|_{1 / 2, \omega, \Gamma} \quad \text { for } \omega \in \mathbb{R}+i \sigma .
$$

Proof. We merely consider the interior problem in $\Omega_{-}$, since the proof for the exterior domain is analogous. Consider the extension $w=\operatorname{ext}_{\Gamma} f$ defined in Lemma 1, and recall that $\|w\|_{1, \omega, \Omega_{-}} \leq$ $C\|f\|_{1 / 2, \omega, \Gamma}$, where $C>0$ is a constant depending only on $\sigma$ and $\Gamma$. It is clear that $v \in H^{1}\left(\Omega_{-}\right)$ solves (18) if and only if $z:=v-w$ satisfies

$$
\begin{cases}\left(\Delta+\omega^{2}\right) z(x)=-\left(\Delta+\omega^{2}\right) w(x), & x \in \Omega_{-}, \\ z(x)=0, & x \in \Gamma .\end{cases}
$$

As in the proof of Proposition 2 one shows that the variational formulation of problem (19) possesses a unique solution $z$ that satisfies $\|z\|_{1, \omega, \Omega} \leq C|\omega|\|w\|_{1, \omega, \Omega}$. This bound implies the claim.

We also need the following result on the injectivity of the interior problem with impedance boundary conditions. 
Lemma 8. The set of $\omega \in \mathbb{C}$ for which there exists non-trivial solutions $w \in H^{1}\left(\Omega_{-}\right)$to

$$
\begin{cases}\left(\Delta+\omega^{2}\right) w(x)=0, & x \in \Omega_{-}, \\ \partial_{n} w(x)+i \omega \alpha w(x)=0, & x \in \Gamma,\end{cases}
$$

is discrete without any point of accumulation.

Proof. Consider the variational formulation associated with $(20): w \in H^{1}\left(\Omega_{-}\right)$solves $(20)$ if and only if

$$
(A(\omega) w, v)_{H^{1}\left(\Omega_{-}\right)}:=\int_{\Omega_{-}}\left[\nabla w \cdot \overline{\nabla v}-\omega^{2} w \bar{v}\right] d x+i \omega \int_{\Gamma} \alpha w \bar{v} d s=0 \text { for all } v \in H^{1}\left(\Omega_{-}\right) .
$$

It is clear from the Rellich compact embedding theorem and trace theorems that the operator $A(\omega)-I d: H^{1}\left(\Omega_{-}\right) \rightarrow H^{1}\left(\Omega_{-}\right)$is compact. Moreover the operator $A(-i)$ is clearly invertible. Since $A(\omega): H^{1}\left(\Omega_{-}\right) \rightarrow H^{1}\left(\Omega_{-}\right)$depends analytically on $\omega$ in $\mathbb{C}$, the result directly follows from the analytic Fredholm theory (see for instance [8]).

For $p \in \mathbb{R}$ and $\sigma>0$, denote

$$
X_{\sigma, \Omega_{ \pm}}^{p}=\left\{u \in H_{\sigma, \Omega_{ \pm}}^{p, 1}: \partial_{t t} u-\Delta u=0 \text { in } \Omega_{ \pm}\right\},
$$

where the differential equation is supposed to hold in the distributional sense. Since we are dealing with Robin boundary conditions, we (formally) introduce the trace operator

$$
\operatorname{tr}_{\Gamma}^{\mathrm{imp}} u=\left(\partial_{n} u-\alpha \partial_{t} u\right)_{\mid \Gamma}
$$

for $u \in X_{\sigma, \Omega_{ \pm}}^{p}$.

Proposition 9. For $p \in \mathbb{R}$ and $\sigma>0$, the operator

$$
\operatorname{tr}_{\Gamma}^{\mathrm{imp}}: X_{\sigma, \Omega_{ \pm}}^{p} \rightarrow H_{\sigma, \Gamma}^{p-1,-1 / 2}
$$

is bounded when $X_{\sigma, \Omega_{ \pm}}^{p}$ is equipped with the canonical norm (9) on $H_{\sigma, \Omega_{ \pm}}^{p, 1}$.

Proof. For $\omega \in \mathbb{R}+i \sigma$, Lemma 1 and a duality argument show that for a Lipschitz domain $\Omega$ and $p \in L^{2}(\Omega)^{3}$ such that $\operatorname{div} p \in L^{2}(\Omega)$ there exists a constant $C$ independent from $\omega$ such that

$$
\left\|\operatorname{tr}_{\Gamma}(p \cdot n)\right\|_{-1 / 2, \omega, \Gamma} \leq C\left(\|p\|_{L^{2}(\Omega)^{3}}+\|\operatorname{div} p /|\omega|\|_{L^{2}(\Omega)}\right) .
$$

Therefore, for $u \in X_{\sigma, \Omega_{+}}^{p}$, using $\Delta \widehat{u}(\omega, \cdot)=-\omega^{2} \widehat{u}(\omega, \cdot)$ in $\Omega_{+}$, we deduce

$$
\left\|\partial_{n} \widehat{u}(\omega, \cdot)\right\|_{-1 / 2, \omega, \Gamma} \leq C\|\widehat{u}(\omega, \cdot)\|_{1, \omega, \Omega_{+}}
$$

where the constant $C$ depends only $\sigma$ and $\Omega_{+}$. Moreover

$$
\|\alpha \omega \widehat{u}(\omega, \cdot)\|_{-1 / 2, \omega, \Gamma} \leq C_{0}|\omega|\|\widehat{u}(\omega, \cdot)\|_{1 / 2, \omega, \Gamma}
$$

where the constant $C_{0}$ depends only $\alpha$ and $\sigma$. Combining these results with Lemma 1 we finally obtain that

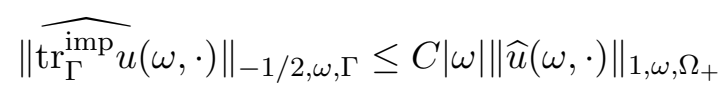

with a (different) constant $C$ depending only $\sigma, \alpha$ and $\Omega_{+}$. The same holds for $\Omega_{-}$. The result of the Lemma then follows from Plancherel's identity. 
Lemma 10. Let $p \in \mathbb{R}$ and $\sigma>0$. If $\zeta \in X_{\sigma, \Omega_{+}}^{p}$, then there exists a sequence $\left(\psi_{n}\right)_{n \in \mathbb{N}}$ in $H_{\sigma, \Gamma_{i}}^{p-2,0}$ such that

$$
L_{\Gamma_{i}}^{\chi} \psi_{n} \stackrel{n \rightarrow \infty}{\longrightarrow} \zeta \text { in } H_{\sigma, \Omega_{+}}^{p-1,1}
$$

Proof. Let $\zeta \in X_{\sigma, \Omega_{+}}^{p}$. Lemma 1 shows that $\operatorname{tr}_{\Gamma} \zeta \in H_{\sigma, \Gamma}^{p, 1 / 2}$, and Proposition 6 implies that there exists a sequence $\left(\psi_{n}\right)_{n \in \mathbb{N}}$ in $H_{\sigma, \Gamma_{i}}^{p-2,0}$ such that

$$
\operatorname{tr}_{\Gamma} L_{\Gamma_{i}}^{\chi}\left(\psi_{n}\right) \stackrel{n \rightarrow \infty}{\longrightarrow} \operatorname{tr}_{\Gamma} \zeta \text { in } H_{\sigma, \Gamma}^{p, 1 / 2}
$$

Note that both $L_{\Gamma_{i}}^{\chi} \psi_{n}$ and $\zeta$ solve the homogeneous wave equation in $\Omega_{+}$. The bounds of Lemma 7 combined with Plancherel's identity imply that

$$
\left\|L_{\Gamma_{i}}^{\chi} \psi_{n}-\zeta\right\|_{H_{\sigma, \Omega_{+}}^{p-1,1}} \leq C\left\|\operatorname{tr}_{\Gamma} L_{\Gamma_{i}}^{\chi}\left(\psi_{n}\right)-\operatorname{tr}_{\Gamma} \zeta\right\|_{H_{\sigma, \Gamma}^{p, 1 / 2}} \stackrel{n \rightarrow \infty}{\longrightarrow} 0
$$

Proposition 11. Let $p \in \mathbb{R}$ and $\sigma>0$. The product $\operatorname{tr}_{\Gamma}^{\mathrm{imp}} L_{\Gamma_{i}}^{\chi}: H_{\sigma, \Gamma_{i}}^{p, 0} \rightarrow H_{\sigma, \Gamma}^{p+1,-1 / 2}$ is bounded injective and has dense range.

Proof. Boundedness follows from the continuity of $L_{\Gamma_{i}}^{\chi}: H_{\sigma, \Gamma_{i}}^{p, 0} \rightarrow H_{\sigma, \Omega_{+}}^{p+2,1}$ (see Proposition 6 ) and and the continuity of $\operatorname{tr}_{\Gamma}^{\mathrm{imp}}: X_{\sigma, \Omega_{ \pm}}^{p+2} \rightarrow H_{\sigma, \Gamma}^{p+1,-1 / 2}$ (see Proposition 9 .

If we assume that $\operatorname{tr}_{\Gamma}^{\mathrm{imp}} L_{\Gamma_{i}}^{\chi} \psi=0$ for some $\psi \in H_{\sigma, \Gamma_{i}}^{p, 0}$, then Lemma 8 implies $\widehat{L_{\Gamma_{i}}^{\chi}}(\omega) \widehat{\psi}(\omega, \cdot)=0$ in $\Omega_{-}$for a.e. $\omega \in \mathbb{R}+i \sigma$. We conclude, as in Proposition 6 , that $\widehat{L_{\Gamma_{i}}}(\omega) \widehat{\psi}(\omega, \cdot)=0$ in $\Omega_{-}$for a.e. $\omega \in \mathbb{R}+i \sigma$. The unique continuation principle then implies $\widehat{L_{\Gamma_{i}}}(\omega) \widehat{\psi}(\omega, \cdot)=0$ in $\mathbb{R}^{3} \backslash \Gamma_{i}$ for a.e. $\omega \in \mathbb{R}+i \sigma$. The second jump relation of (49) finally shows that $\psi=0$.

To prove denseness of the range of $\operatorname{tr}_{\Gamma}^{\mathrm{imp}} L_{\Gamma_{i}}^{\chi}$, consider $\zeta \in H_{\sigma, \Gamma}^{p+1,-1 / 2}$. Since the embedding $H_{\sigma, \Gamma}^{p+3,1 / 2}$ into $H_{\sigma, \Gamma}^{p+1,-1 / 2}$ is dense (which can be easily observed using a cut-off argument in the frequency domain and the dense embedding of $H^{1 / 2}(\Gamma)$ into $H^{-1 / 2}(\Gamma)$ ), there exists a sequence $\left(\zeta_{n}\right)_{n \in \mathbb{N}} \subset H_{\sigma, \Gamma}^{p+3,1 / 2}$ such that $\zeta_{n} \rightarrow \zeta$ in $H_{\sigma, \Gamma}^{p+1,-1 / 2}$. Due to Proposition 2 , there exists $u_{n} \in H_{\sigma, \Omega_{+}}^{p+2,1}$ such that

$$
\begin{cases}\partial_{t t} u_{n}(t, x)-\Delta u_{n}(t, x)=0, & t \in \mathbb{R}, x \in \Omega_{+}, \\ \partial_{n} u_{n}(t, x)-\alpha \partial_{t} u_{n}(t, x)=\zeta_{n}, & t \in \mathbb{R}, x \in \Gamma\end{cases}
$$

Proposition 10 states that we can approximate the $u_{n}$ by potentials $X_{\sigma, \Omega_{+}}^{p+2} \ni L_{\Gamma_{i}}^{\chi} \psi_{n, m} \stackrel{m \rightarrow \infty}{\longrightarrow} u_{n}$ in $H_{\sigma, \Omega_{+}}^{p+2,1}$, where $\left(\psi_{n, m}\right)_{m \in \mathbb{N}} \subset H_{\sigma, \Gamma}^{p, 0}$. Finally, the continuity of $\operatorname{tr}_{\Gamma}^{\text {imp }}$ from $X_{\sigma, \Omega_{+}}^{p+2}$ into $H_{\sigma, \Gamma}^{p+1,-1 / 2}$ shows that

$$
\operatorname{tr}_{\Gamma}^{\mathrm{imp}} L_{\Gamma_{i}}^{\chi} \psi_{n, m} \stackrel{m \rightarrow \infty}{\longrightarrow} \operatorname{tr}_{\Gamma}^{\mathrm{imp}} u_{n}=\zeta_{n} \quad \text { in } H_{\sigma, \Gamma}^{p+1,-1 / 2} .
$$

Since, by construction, $\zeta_{n} \stackrel{n \rightarrow \infty}{\longrightarrow} \zeta$ in $H_{\sigma, \Gamma}^{p+1,-1 / 2}$ the proof is finished.

Recall now the definition of the solution operator $G$ from $(10)$. For $\sigma>0$ and $p \in \mathbb{R}$ we introduce a restriction of this operator to $\Gamma_{m}$,

$$
G_{\Gamma_{m}}: H_{\sigma, \Gamma}^{p+1,-1 / 2} \rightarrow H_{\sigma, \Gamma_{m}}^{p, 1 / 2} \quad \text { defined by } G_{\Gamma_{m}}(g)=\operatorname{tr}_{\Gamma_{m}} G(g) .
$$

Proposition 4 and Lemma 1 ensure that this operator is well-defined and bounded. 
Proposition 12. Let $p \in \mathbb{R}$ and $\sigma>0$. Then the operator $G_{\Gamma_{m}}: H_{\sigma, \Gamma}^{p+1,-1 / 2} \rightarrow H_{\sigma, \Gamma_{m}}^{p, 1 / 2}$ is injective with dense range.

Proof. Let $u=G(g)$ for $g \in H_{\sigma, \Gamma}^{p+1,-1 / 2}$ (see 10 for a definition of the solution operator $G$ ). Assume that $G_{\Gamma_{m}}(g)=0$. Then, due to our assumptions on $\Gamma_{m}$ that is either a closed Lipschitz surface or an analytic open surface, the unique continuation property and unique solvability of exterior scattering problems at complex frequencies in $\mathbb{R}+i \sigma$ imply that $\widehat{u}(\omega, \cdot)=0$ in $\Omega_{+}$for a.e. $\omega \in \mathbb{R}+i \sigma$. This implies that $g=\operatorname{tr}_{\Gamma}^{\mathrm{imp}} u=0$.

The denseness of the range of $G_{\Gamma_{m}}$ can be obtained by observing that the range of $G_{\Gamma_{m}}$ contains $\operatorname{tr}_{\Gamma_{m}} u$ where

$$
u(t, x)=\left(L_{\Gamma} \psi\right)(t, x)=\int_{\Gamma} \frac{\psi(t-|x-y|, y)}{4 \pi|x-y|} d \sigma_{y}
$$

for $t>0$ and $x \in \Omega_{+}$and for some density $\psi \in H_{\sigma, \Gamma}^{p+3,-1 / 2}$. This simply comes from the fact that, by the boundary jumps 52 ,

$$
\operatorname{tr}_{\Gamma}^{\mathrm{imp}} u=\left(-\frac{1}{2} I+K_{\Gamma}\right) \psi-\alpha S_{\Gamma} \partial_{t} \psi=: g
$$

and from Proposition $19 g \in H_{\sigma, \Gamma}^{p+1,-1 / 2}$. Consequently, the range of $G_{\Gamma_{m}}$ contains $\operatorname{tr}_{\Gamma_{m}} L_{\Gamma}\left(H_{\sigma, \Gamma}^{p+3,-1 / 2}\right)$. Following the lines of last part of the proof of Proposition 6, one can show that

$$
\operatorname{tr}_{\Gamma_{m}} L_{\Gamma}: H_{\sigma, \Gamma}^{p+3,-1 / 2} \rightarrow H_{\sigma, \Gamma_{m}}^{p, 1 / 2}
$$

has dense range. This concludes the proof.

\section{A Theoretical Result Motivating Time Domain Sampling Algo- rithms}

As already explained in the introduction, the inverse problem we consider is to reconstruct the obstacle $\Omega_{-}$from time domain near-field measurements of scattered waves. We use incident pulses in the form of convolutions in time of the fundamental solution of the wave equation with a pulse $\chi$

$$
u^{i}(t, x ; y):=\frac{\chi(t-|x-y|)}{4 \pi|x-y|}, \quad t \in \mathbb{R}, x \in \mathbb{R}^{3} \backslash\{y\}
$$

The scattered field $u(\cdot, \cdot ; y)$ solves the scattering problem $(2)$ for boundary data $g=\operatorname{tr}_{\Gamma}^{\mathrm{imp}} u^{i}(\cdot, \cdot ; y)$ on $\Gamma$.

The inverse problem we consider is to reconstruct $\Omega_{-}$from the partial knowledge of the scattered waves

$$
\left\{u(t, x ; y): t \in \mathbb{R}, x \in \Gamma_{m}, y \in \Gamma_{i}\right\} .
$$

We assume that $\Gamma_{i, m}$ and $\chi$ satisfy the same hypothesis as in Section 2.3 (see the beginning of that section for details). Since $y \in \Gamma_{i}$ and due to (12) we infer that

$$
\operatorname{tr}_{\Gamma}^{\mathrm{imp}} u^{i}=\partial_{n} u^{i}-\alpha \partial_{t} u^{i} \in H_{\sigma, \Gamma}^{0,-1 / 2}
$$


In consequence, Proposition 4 implies that the scattered field $u(\cdot, \cdot ; y)$ is well-defined in $H_{\sigma, \Omega_{+}}^{-1,1}$ and the trace theorem 1 implies that $\operatorname{tr}_{\Gamma_{m}} u(\cdot, \cdot ; y)$ is well-defined in $H_{\sigma, \Gamma_{m}}^{-1,1 / 2}$. Since $\operatorname{tr}_{\Gamma_{m}} u(\cdot, \cdot ; y)=$ $-G_{\Gamma_{m}} \operatorname{tr}_{\Gamma}^{\mathrm{imp}}\left(u^{i}\right)$, the linear combination of several incident pulses produces the corresponding linear combination of the measurements. Therefore, for regular densities $\psi$ the near-field operator $N$ simply satisfies

$$
\begin{aligned}
(N \psi)(t, x) & =\int_{\mathbb{R}} \int_{\Gamma_{i}} u(t-\tau, x ; y) \psi(\tau, y) d y d \tau \\
& =-G_{\Gamma_{m}} \operatorname{tr}_{\Gamma}^{\operatorname{imp}}\left(\int_{\mathbb{R}} \int_{\Gamma_{i}} u^{i}(\cdot-\tau, \cdot ; y) \psi(\tau, y) d y d \tau\right)(t, x) \\
& =-G_{\Gamma_{m}} \operatorname{tr}_{\Gamma}^{\mathrm{imp}} L_{\Gamma_{i}}^{\chi} \psi(t, x), \quad(t, x) \in \mathbb{R} \times \Gamma_{m} .
\end{aligned}
$$

We therefore deduce the following (mapping) properties of $N$.

Proposition 13. Let $\sigma>0$ and $p \in \mathbb{R}$. Then the operator $N$ is bounded, injective and has dense range from $H_{\sigma, \Gamma_{i}}^{p, 0}$ to $H_{\sigma, \Gamma_{m}}^{p, 1 / 2}$.

Proof. This follows from the factorization $N=-G_{\Gamma_{m}} \operatorname{tr}_{\Gamma}^{\mathrm{imp}} L_{\Gamma_{i}}^{\chi}$ and Propositions 11 and 12 .

With the notation $L_{\sigma, \Gamma}^{2}=H_{\sigma, \Gamma_{i}}^{0,0}$, this result in particular implies that for all $\sigma>0$,

$$
N: L_{\sigma, \Gamma_{i}}^{2} \rightarrow L_{\sigma, \Gamma_{m}}^{2}
$$

is bounded and injective with dense range.

We now introduce test functions as traces of monopole or dipole solutions to the wave equation, convolved with a function $\varsigma \in H^{1}(\mathbb{R})$ with compact support. (We use $H^{1}$-regular pulses since we will consider derivatives of the corresponding waves.) For a point $z \in \mathbb{R}^{3} \backslash \Gamma_{m}$ and $\tau \in \mathbb{R}$ we set

$$
\phi_{z}(t, x):=\frac{\varsigma(t-\tau-|x-z|)}{4 \pi|x-z|}, \quad(t, x) \in \mathbb{R} \times \Gamma_{m}
$$

to define monopole test functions, and using a direction $d \in \mathbb{S}^{2}$, we define dipole test functions by

$$
\phi_{z}^{d}(t, x):=d \cdot \nabla_{x} \frac{\varsigma(t-\tau-|x-z|)}{4 \pi|x-z|}, \quad(t, x) \in \mathbb{R} \times \Gamma_{m} .
$$

The dependence of these functions on $\tau$ is not denoted explicitly, since this parameter will be fixed in all later computations.

The main principle of the linear sampling method is to first approximately solve the so-called near field equation $N g=\phi_{z}$ (or $N g=\phi_{z}^{d}$ for the dipole test functions) for the unknown $g=g_{z}$, for many points $z$ on a sampling grid. Theorem 14 below indicates that the norm of $g_{z}$ is large when $z$ is outside the scatterer $\Omega_{-}$. Hence, in a second step, one gets an image of the scatterer by plotting $z \mapsto 1 /\left\|g_{z}\right\|$ on the sampling grid.

In the following main theorem $\ell_{z}$ refers to either the monopole test functions $\phi_{z}$ or the dipole test functions $\phi_{z}^{d}$ for a fixed dipole direction $d$.

Theorem 14. Let $\sigma>0, \tau \in \mathbb{R}$, and $d \in \mathbb{S}^{2}$. 
1. For $z \in \Omega_{-}$and $\epsilon>0$ there exists $g_{z, \epsilon} \in L_{\sigma, \Gamma_{i}}^{2}$ such that

$$
\lim _{\epsilon \rightarrow 0}\left\|\operatorname{tr}_{\Gamma}^{\mathrm{imp}} L_{\Gamma_{i}}^{\chi} g_{z, \epsilon}\right\|_{H_{\sigma, \Gamma}^{1,-1 / 2}}<\infty, \quad \lim _{z \rightarrow \partial \Omega_{-}}\left\|g_{z, \epsilon}\right\|_{L_{\sigma, \Gamma_{i}}^{2}}=\infty, \text { and } \lim _{\epsilon \rightarrow 0}\left\|N g_{z, \epsilon}-\ell_{z}\right\|_{L_{\sigma, \Gamma_{m}}^{2}}=0
$$

2. For $z \in \mathbb{R}^{3} \backslash\left(\Omega_{-} \cup \Gamma_{m}\right)$ and for all $g_{z, \epsilon} \in L_{\sigma, \Gamma_{i}}^{2}$ such that $\lim _{\epsilon \rightarrow 0}\left\|N g_{z, \epsilon}-\ell_{z}\right\|_{L_{\sigma, \Gamma_{m}}^{2}}=0$ it holds that

$$
\lim _{\epsilon \rightarrow 0}\left\|\operatorname{tr}_{\Gamma}^{\mathrm{imp}} L_{\Gamma_{i}}^{\chi} g_{z, \epsilon}\right\|_{H_{\sigma}^{1,-1 / 2}}=\infty, \quad \text { and } \quad \lim _{\epsilon \rightarrow 0}\left\|g_{z, \epsilon}\right\|_{L_{\sigma, \Gamma_{i}}^{2}}=\infty .
$$

The function $g_{z, \epsilon}$ can be chosen as the unique minimizer of the Tikhonov functional

$$
g \mapsto\left\|N g-\ell_{z}\right\|_{L_{\sigma, \Gamma_{m}}^{2}}^{2}+\epsilon\|g\|_{L_{\sigma, \Gamma_{i}}^{2}}^{2} .
$$

Proof. Let us recall the factorization $N=-G_{\Gamma_{m}} \operatorname{tr}_{\Gamma}^{\mathrm{imp}} L_{\Gamma_{i}}^{\chi}$ of the near-field operator, where the (regularized) single-layer potential $L_{\Gamma_{i}}^{\chi}$ was defined in (11), the operator $\operatorname{tr}_{\Gamma}^{\text {imp }}$ is defined by (22) and the operator $G_{\Gamma_{m}}$ is defined by (23).

1. Let $z \in \Omega_{-}$. To simplify our notation, we will extend the definition of the test function $\ell_{z}$ to all of $\mathbb{R}^{3} \backslash\{z\}$ by the explicit formula (27) or (28). Our assumption that $z \in \Omega_{-}$implies that

$$
\operatorname{Grr}_{\Gamma}^{\operatorname{imp}}\left(\ell_{z}\right)=-\ell_{z}
$$

since $\ell_{z}$ is the unique causal solution to the wave equation with Robin boundary data $-\operatorname{tr}_{\Gamma}^{\operatorname{imp}}\left(\ell_{z}\right)$. Therefore

$$
-G_{\Gamma_{m}} \operatorname{tr}_{\Gamma}^{\mathrm{imp}}\left(\ell_{z}\right)=\ell_{z} \quad \text { on } \mathbb{R} \times \Gamma_{m}
$$

Due to Proposition 11 (with $p=0$ ), we can approximate $\operatorname{tr}_{\Gamma}^{\mathrm{imp}} \ell_{z} \in H_{\sigma, \Gamma}^{1,-1 / 2}$ by Robin traces of layer potentials: for $\epsilon>0$ there exists $g_{z, \epsilon} \in L_{\sigma, \Gamma_{i}}^{2}$ such that

$$
\left\|\operatorname{tr}_{\Gamma}^{\mathrm{imp}} L_{\Gamma_{i}}^{\chi} g_{z, \epsilon}-\operatorname{tr}_{\Gamma}^{\mathrm{imp}} \ell_{z}\right\|_{H_{\sigma, \Gamma}^{1,-1 / 2}} \leq \epsilon .
$$

The continuity of $G_{\Gamma_{m}}$ from $H_{\sigma, \Gamma}^{1,-1 / 2}$ into $H_{\sigma, \Gamma_{m}}^{0,1 / 2}$ (see Proposition 12 implies that

$$
\left\|N g_{z, \epsilon}-\ell_{z}\right\|_{H_{\sigma, \Gamma m}^{0,0}} \leq C\left\|N g_{z, \epsilon}-\ell_{z}\right\|_{H_{\sigma, \Gamma_{m}}^{0,1 / 2}} \leq C\left\|\operatorname{tr}_{\Gamma}^{\mathrm{imp}} L_{\Gamma_{i}}^{\chi} g_{z, \epsilon}-\operatorname{tr}_{\Gamma}^{\mathrm{imp}} \ell_{z}\right\|_{H_{\sigma, \Gamma}^{1,-1 / 2}} \leq C \epsilon
$$

which obviously implies that $\lim _{\epsilon \rightarrow 0}\left\|N g_{z, \epsilon}-\ell_{z}\right\|_{L_{\sigma, \Gamma m}^{2}}=0$.

Let us now show that $\lim _{z \rightarrow \Gamma}\left\|g_{z, \epsilon}\right\|_{L_{\sigma, \Gamma_{i}}^{2}}=\infty$. We argue by contradiction, and assume that there is a sequence $\left\{z_{n}, n \in \mathbb{N}\right\} \subset \Omega_{-}$such that $z_{n} \rightarrow z \in \partial \Omega_{-}$and such that

$$
\left\|g_{z_{n}, \epsilon}\right\|_{H_{\sigma, \Omega_{-}}^{2,1}} \leq C
$$

for some constant $C>0$. Then it holds that

$$
\left\|\operatorname{tr}_{\Gamma}^{\mathrm{imp}} \ell_{z_{n}}\right\|_{H_{\sigma, \Gamma}^{1,-1 / 2}} \leq\left\|\operatorname{tr}_{\Gamma}^{\mathrm{imp}} L_{\Gamma_{i}}^{\chi} g_{z, \epsilon}-\operatorname{tr}_{\Gamma}^{\mathrm{imp}} \ell_{z}\right\|_{H_{\sigma, \Gamma}^{1,-1 / 2}}+\left\|\operatorname{tr}_{\Gamma}^{\mathrm{imp}} L_{\Gamma_{i}}^{\chi} g_{z, \epsilon}\right\|_{H_{\sigma, \Gamma}^{1,-1 / 2}} \leq C^{\prime}(\epsilon+C),
$$

where we exploited the boundedness of $\operatorname{tr}_{\Gamma}^{\mathrm{imp}} L_{\Gamma_{i}}^{\chi}$ shown in Proposition 11. Additionally,

$$
\left\|\operatorname{tr}_{\Gamma}^{\mathrm{imp}} \ell_{z_{n}}\right\|_{H_{\sigma, \Gamma}^{1,-1 / 2}}^{2} \geq\left\|\ell_{z_{n}}\right\|_{H_{\sigma, \Omega_{+}}^{0,1}}^{2}=\int_{\infty-i \sigma}^{\infty+i \sigma}\left\|\widehat{\ell_{z_{n}}}(\omega, \cdot)\right\|_{1, \omega, \Omega_{+}}^{2} d \omega
$$


due to (31) and Proposition 2. Since $\ell_{z_{n}}$ is the convolution in time of $\varsigma(\cdot-\tau)$ and the fundamental solution to the wave equation or to its derivative, we obtain that

$$
\left\|\operatorname{tr}_{\Gamma}^{\mathrm{imp}} \ell_{z_{n}}\right\|_{H_{\sigma, \Gamma}^{1,-1 / 2}}^{2} \geq \int_{-\infty+i \sigma}^{\infty+i \sigma}\left|e^{-i \tau \omega} \widehat{\varsigma}(\omega)\right|^{2}\left\|\Psi_{\omega}\left(\cdot-z_{n}\right)\right\|_{1, \omega, \Omega_{+}}^{2} d \omega
$$

where $\Psi_{\omega}(x)=\Phi_{\omega}(x)$ or $\Psi_{\omega}(x)=d \cdot \nabla \Phi_{\omega}(x)$ and $\Phi_{\omega}(x):=\exp (i \omega|x|) / 4 \pi|x|$. One can easily check that $\left\|\Psi_{\omega}\left(\cdot-z_{n}\right)\right\|_{1, \omega, \Omega_{+}} \rightarrow \infty$ as $z_{n} \rightarrow z$ (see, e.g., [17]). By definition, $\varsigma$ has compact support so that the Laplace transform $\widehat{\varsigma}$ vanishes at most on a discrete set of points. Now we can conclude using Fatou's lemma that

$$
\liminf _{n \rightarrow \infty}\left\|\operatorname{tr}_{\Gamma}^{\mathrm{imp}} \ell_{z_{n}}\right\|_{H_{\sigma, \Gamma}^{1,-1 / 2}}^{2} \geq \int_{-\infty+i \sigma}^{\infty+i \sigma} \mid e^{-\left.i \tau \omega \widehat{\varsigma}(\omega)\right|^{2}} \liminf _{n \rightarrow \infty}\left\|\Psi_{\omega}\left(\cdot-z_{n}\right)\right\|_{1, \omega, \Omega_{+}}^{2} d \omega=\infty
$$

which is a contradiction to 33 .

2. Let now $z \in \mathbb{R}^{3} \backslash\left(\Omega_{-} \cup \Gamma_{m}\right)$. Since the range of $N$ is dense in $L_{\sigma, \Gamma_{i}}^{2}$, it is a well-known result on Tikhonov regularization that the minimizer $g_{z, \epsilon}$ to 30 satisfies

$$
\lim _{\epsilon \rightarrow 0}\left\|N g_{z, \epsilon}-\ell_{z}\right\|_{L_{\sigma, \Gamma m}^{2}}=0 \text {. }
$$

It remains to show that every sequence verifying (34) is such that

$$
\lim _{\epsilon \rightarrow 0}\left\|\operatorname{tr}_{\Gamma}^{\mathrm{imp}} L_{\Gamma_{i}}^{\chi} g_{z, \epsilon}\right\|_{H_{\sigma, \Gamma}^{1,-1 / 2}}=\infty
$$

Choose a positive zero sequence $\left(\epsilon_{n}\right)_{n \in \mathbb{N}}$ and suppose that there exists $C>0$ such that

$$
\left\|\operatorname{tr}_{\Gamma}^{\mathrm{imp}} L_{\Gamma_{i}}^{\chi} g_{z, \epsilon_{n}}\right\|_{H_{\sigma, \Gamma}^{1,-1 / 2}} \leq C .
$$

Hence, there is a weakly convergent subsequence $v_{n}:=\operatorname{tr}_{\Gamma}^{\mathrm{imp}} L_{\Gamma_{i}}^{\chi} g_{z, \epsilon_{n}}$ that weakly converges in $H_{\sigma, \Gamma}^{1,-1 / 2}$ to some $v \in H_{\sigma, \Gamma}^{1,-1 / 2}$. (By abuse of notation we omit to denote this subsequence explicitly.) Let us set

$$
w=G v \in L_{\sigma}^{2}\left(\mathbb{R}, H^{1}\left(\Omega_{+}\right)\right) .
$$

Since $v_{n} \rightarrow v$ weakly in $H_{\sigma, \Gamma}^{1,-1 / 2}$, the factorization of $N$ implies that $N g_{z, \epsilon_{n}} \rightarrow \operatorname{tr}_{\Gamma_{m}} w$ in $L_{\sigma, \Gamma_{m}}^{2}$ as $n \rightarrow \infty$. In consequence, (34) implies that $w=\ell_{z}$ on $\mathbb{R} \times \Gamma_{m}$, which means that the Laplace transforms of both functions coincide:

$$
\widehat{w}(\omega, \cdot)=\widehat{\ell}_{z}(\omega, \cdot) \text { in } L^{2}\left(\Gamma_{m}\right) \text {, for a.e. } \omega \in \mathbb{R}+i \sigma .
$$

Both $\widehat{w}(\omega, \cdot)$ and $\widehat{\ell}_{z}(\omega, \cdot)$ satisfy the Helmholtz equation with complex frequency $\omega$ in $\Omega_{+} \backslash\{z\}$. Due to our assumptions on $\Gamma_{m}$ that is either a closed Lipschitz surface or an analytic open surface, the unique continuation property and unique solvability of exterior scattering problems at complex frequencies in $\mathbb{R}+i \sigma$ imply that $\widehat{w}(\omega, \cdot)=\widehat{\ell}_{z}(\omega, \cdot)$ in $H^{1}\left(\Omega_{+} \backslash\{z\}\right)$ for a.e. $\omega \in \mathbb{R}+i \sigma$. However, as explained before, $x \mapsto \hat{\ell}_{z}(\omega, x)$ fails to be $H^{1}$ in a neighborhood of $z$. This contradiction shows that our assumption (35) was wrong and concludes the proof. 


\section{Fast Implementation and Numerical Experiments}

In this section, we demonstrate that the linear sampling algorithm (see the description before the main Theorem 14) is able to reconstruct obstacles with mixed boundary conditions of Dirichlet, Neumann or Robin-type. We start by sketching the algorithm. Several aspects of the technique are explained afterwards in more details. Finally, we present some numerical results that are, for the sake of computational simplicity, two-dimensional. Extending the above theoretical analysis to dimension two is possible since all important arguments stem from Laplace transform techniques. The special form of the fundamental solution of the wave equation in two dimensions, however, then yields more complicated expressions compared to the three-dimensional case.

Inversion algorithm. A centered second-order finite-difference scheme with perfectly matched layers on the boundary of the computational domain provides us with numerical approximations to the scattering data

$$
u\left(n \Delta t, x_{m} ; y_{i}\right), \quad 1 \leq n \leq N_{T}=\lfloor T / \Delta t\rfloor, 1 \leq m \leq N_{m}, 1 \leq i \leq N_{i},
$$

for points $y_{i} \in \Gamma_{i}, x_{m} \in \Gamma_{m}$, and a time step $\Delta t$ (for details on the numerical scheme we use to compute these approximations, see the appendix in [16]). Using this data we discretize the near-field operator from $(26)$ as a discrete convolution operator $\mathrm{N}: \mathbb{R}^{N_{T} \times N_{i}} \rightarrow \mathbb{R}^{N_{T} \times N_{m}}$ by

$$
(\mathrm{Ng})(n, m):=\sum_{i=1}^{N_{i}} \sum_{j=0}^{n-1} u\left((n-j) \Delta t, x_{m} ; y_{i}\right) \mathrm{g}(i, j), \quad n=1, \ldots, N_{T}, m=1, \ldots, N_{m} .
$$

The test functions for the method are discretized analogously by point-evaluations, e.g., the discretization of the monopoles $\phi_{z}$ and the dipoles $\phi=\phi_{z}^{d}$ from 27 is $\phi_{z} \in \mathbb{R}^{N_{T} \times N_{m}}$ and $\phi_{z}^{d} \in$ $\mathbb{R}^{N_{T} \times N_{m}}$, respectively,

$$
\phi_{z}(n, m)=\phi_{z}\left(n \Delta t, x_{m}\right), \quad \phi_{z}^{d}(n, m)=\phi_{z}^{d}\left(n \Delta t, x_{m}\right) .
$$

For simplicity, we denote the two canonical unit vectors in $\mathbb{R}^{2}$ by $e_{1}=(1,0)^{\top}$ and $e_{2}=(0,1)^{\top}$.

The numerical inversion is done along the following scheme:

1. Compute the $M$ largest singular values $\left(\sigma_{i}\right)_{1 \leq i \leq M}$ of $\mathrm{N}$ and their corresponding left and right vectors $\left(\mathrm{u}_{i}\right)_{1 \leq i \leq M}$ and $\left(\mathrm{v}_{i}\right)_{1 \leq i \leq M}$, respectively. The computation of the truncated singular value decomposition is done using only matrix-vector multiplication and the (highdimensional) matrix $\mathrm{N}$ is never set up. The evaluation of $\mathrm{N}$ is coded using the fast Fourier transform (FFT), see below. Typically, one chooses $M$ such that $\sigma_{M} / \sigma_{1}$ is smaller than the noise level.

2. Choose an appropriate shift in time $\tau$ and sampling points $z$ in a regular sampling grid of the probed region. Evaluate the Tikhonov regularized solutions $\mathrm{g}_{z, \epsilon}, \mathrm{g}_{z, \epsilon}^{e_{1}}$ and $\mathrm{g}_{z, \epsilon}^{d}$ to the equations $\mathrm{Ng}=\phi_{z}, \mathrm{Ng}=\phi_{z}^{e_{1}}$ and $\mathrm{Ng}=\phi_{z}^{e_{2}}$, respectively. The explicit formula for $\mathrm{g}_{z, \epsilon}$ is

$$
\mathrm{g}_{z, \epsilon}=\sum_{i=1}^{M} \frac{\sigma_{i}\left(\phi_{z}, \mathrm{u}_{i}\right)_{\mathbb{R}^{N_{T} \times N_{m}}}}{\epsilon^{2}+\sigma_{i}^{2}} \mathrm{v}_{i}
$$

and formulas for $\mathrm{g}_{z, \epsilon}^{e_{1,2}}$ can be derived analogously. 
3. Assemble the Gram matrix $A_{z, \epsilon}$ of $\mathrm{g}_{z, \epsilon}^{e_{1}}$ and $\mathrm{g}_{z, \epsilon}^{e_{2}}$ and compute its largest eigenvalue $\lambda_{\epsilon}(z)$ (see the next paragraph for details). The three obstacle indicators we consider are

$$
G_{\epsilon}^{(0)}(z)=\left\|\mathrm{g}_{z, \epsilon}\right\|, \quad G_{\epsilon}^{(1)}(z)=\sqrt{\lambda_{\epsilon}(z)}
$$

and

$$
G^{(\max )}(z)=\max \left(\left\|G_{\epsilon}^{(0)}\right\|_{\infty} / G_{\epsilon}^{(1)}(z),\left\|G_{\epsilon}^{(1)}\right\|_{\infty} / G_{\epsilon}^{(1)}(z)\right)
$$

for points $z$ in the sampling region.

Reconstruction via dipole testing. Despite the theoretical statement of Theorem 14 does not distinguish between monopole or dipole test functions, numerical experiments show that the reconstruction quality for different types of obstacles depends nevertheless strongly on this choice. While the monopole test functions from (27) are better suited to reconstruct Dirichlet obstacles, Neumann obstacles typically are better detected by the dipole test functions from (28). In the latter case, one needs to compute regularized solutions to the near-field equation

$$
N g_{z}^{\nu}=\phi_{z}^{\nu}
$$

for a given dipole direction $\nu \in \mathbb{S}^{1}$. We propose to search for a polarization $\nu$ maximizing $\left\|g_{z}^{\nu}\right\|$, and define

$$
G^{(1)}(z)=\max _{\nu \in \mathbb{S}^{1}}\left\|g_{z}^{\nu}\right\|
$$

For simplicity, we omit to denote the noise level $\epsilon$ explicitly in this section. By linearity, it is clear that

$$
G^{(1)}(z)=\max _{\nu \in \mathbb{S}^{1}}\left\|\nu \cdot\left(g_{z}^{e_{1}}, g_{z}^{e_{2}}\right)^{T}\right\|
$$

where $g_{z}^{e_{1}}$ and $g_{z}^{e_{2}}$ are the solutions of (40) associated to the dipole directions $e_{1}$ and $e_{2}$, respectively. If we set $\nu=\left(\nu_{1}, \nu_{2}\right)^{T}$, then one gets

$$
\left\|\nu \cdot\left(g_{z}^{e_{1}}, g_{z}^{e_{2}}\right)^{T}\right\|^{2}=\left|\nu_{1}\right|^{2}\left\|g_{z}^{e_{1}}\right\|^{2}+\left|\nu_{2}\right|^{2}\left\|g_{z}^{e_{2}}\right\|+2 \nu_{1} \nu_{2}\left(g_{z}^{e_{1}}, g_{z}^{e_{2}}\right)=\left(A_{z} \nu, \nu\right)
$$

where

$$
A_{z}=\left(\begin{array}{cc}
\left\|g_{z}^{e_{1}}\right\|^{2} & \left(g_{z}^{e_{1}}, g_{z}^{e_{2}}\right) \\
\left(g_{z}^{e_{1}}, g_{z}^{e_{2}}\right) & \left\|g_{z}^{e_{2}}\right\|^{2}
\end{array}\right)
$$

is the Gram matrix of $\left(g_{z}^{e_{1}}, g_{z}^{e_{2}}\right)$. Thus

$$
G^{(1)}(z)=\sqrt{\lambda(z)}
$$

where $\lambda(z)$ is the greatest eigenvalue of $A_{z}$. In the finite-dimensional discrete setting, $g_{z}^{e_{1,2}}$ is replaced by $\mathrm{g}_{z}^{e_{1,2}}$, yielding $G^{(1)}=\sqrt{\lambda}$ for $\lambda(z)$ the largest eigenvalue of the corresponding Gram matrix. 
Convolution using the fast Fourier transform. To compute singular vectors and values of the finite-dimensional convolution operator $\mathrm{N}$ we use ARPACK routines implementing an implicitly restarted Arnoldi iteration that avoid the computation of the matrix representation of $\mathrm{N}$. (The dimension of the matrix is $N_{T} N_{m} \times N_{T} N_{i}$ and hence too large to store it in the memory.) Then, however, the efficient evaluation of a matrix-vector-product $\mathrm{g} \mapsto \mathrm{Ng}$ is crucial. The evaluation via (36) costs $N_{T}^{2} N_{m} N_{i}$ operations. Instead, we exploit the convolution structure of $\mathrm{N}$ and use FFT routines implemented in the FFTW library [12. To this end, we rewrite the convolution $\sum_{j=0}^{n-1} u\left((n-j) \Delta t, x_{m} ; y_{i}\right) \mathrm{g}(i, j)$ as a circular convolution. This is achieved by extending the arrays $u\left(n \Delta t, x_{m} ; y_{i}\right)_{n=1}^{N_{T}}$ and $\mathrm{g}(n, i)_{n=1}^{N_{T}}$ by zero to arrays of length $2 N_{T}-1$. The circular convolution of these two extended arrays is the inverse discrete Fourier transform of the componentwise product of the discrete Fourier transforms of the extended arrays. Since the discrete Fourier transforms can be computed in $O\left(N_{T} \log \left(N_{T}\right)\right)$ operations, the cost to evaluate one matrix-vector product $\mathrm{g} \mapsto \mathrm{Ng}$ reduces to $O\left(N_{T} \log \left(N_{T}\right) N_{m} N_{i}\right)$ operations, which is order-optimal in $N_{T}$ up to logarithmic terms. Computing the discrete Fourier transform using FFTW routines is most efficient if the length of the transformed vectors possesses only small prime factors. For this reason we throw away a few of the last wave measurements (that are anyway close to zero) to arrive at a convenient FFT length factoring in prime numbers less than or equal to 13 . Implementing the evaluation $\mathrm{g} \mapsto \mathrm{Ng}$ using the FFT speeds up the computation of truncated singular systems by a factor larger than 16 for typical problem sizes. Direct implementations of (36) can lead to computations of singular systems that take several hours, while the FFT-based version takes several minutes (see also the computation times detailed in the next paragraph).

Numerical results. In all our numerical examples we use equidistant emitters and receivers placed on the boundary of a square with side length 5 around the sampling region. The sampling region itself is a centered square of side length 4 . Note that restricting the sampling region to a subset enclosed by the emitters and receivers does not require a-priori knowledge, at least if the receivers surround all the obstacle: Since we know that the background wave speed equals one, it would be easy to even reconstruct the convex hull of the scattering object just by considering arrival times of the scattered fields at the emitters. In some sense, the aim of the method we consider is to find more geometric information than the convex hull.

For all experiments we use the pulse $\chi(t)=\left[\sin (4 t) \exp \left(-1.6(t-3)^{2}\right)\right]^{\prime}, t>0$, to generate incident fields $u^{i}$ of the form (25). Figure 4 shows the pulse and its wave number spectrum. In
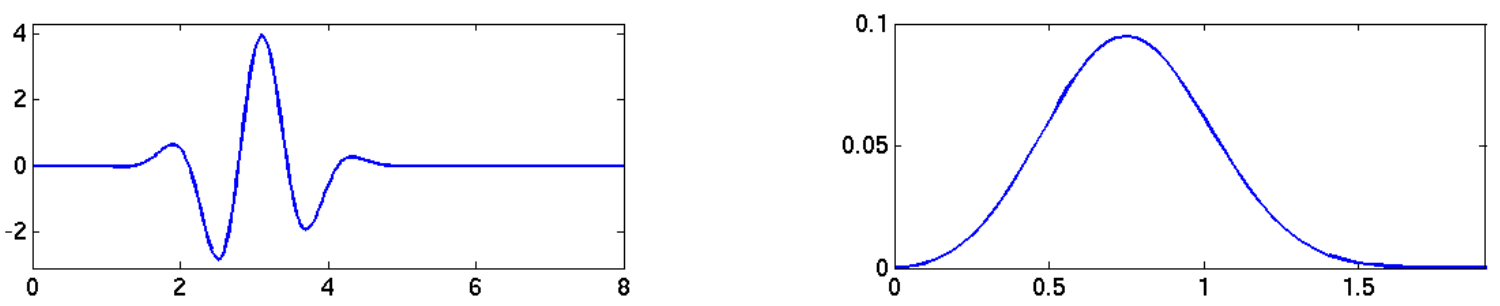

Figure 2: On the left: The pulse $t \mapsto \chi(t)$. On the right: the frequency spectrum $\omega \mapsto|\hat{\chi}(\omega)|$ of the pulse $\chi$.

particular, we note that the maximum of the spectrum is roughly at $\omega=0.76$. The corresponding 
wave length and wave number are $\lambda=1.3$ and $k=4.8$, respectively.

All computations that we present below were done on an Intel Xeon $3.20 \mathrm{GHz}$ processor with 12 GB memory without using multi-threading or parallel computing techniques.

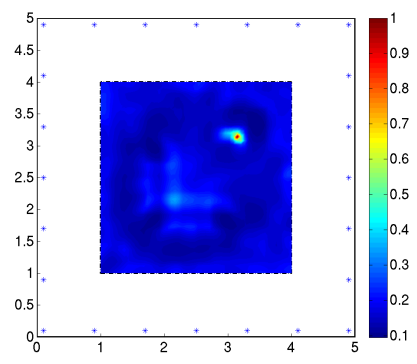

(a)

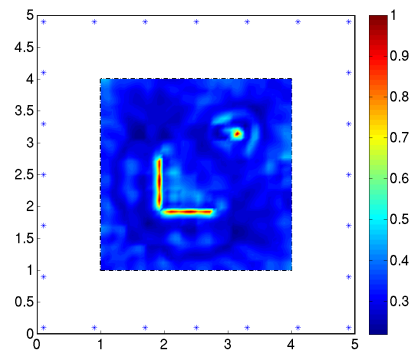

$(d)$

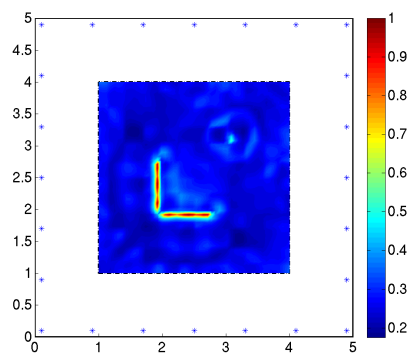

(b)

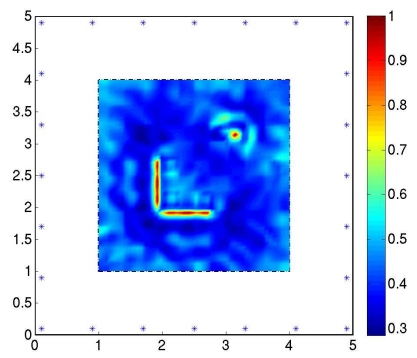

(e)

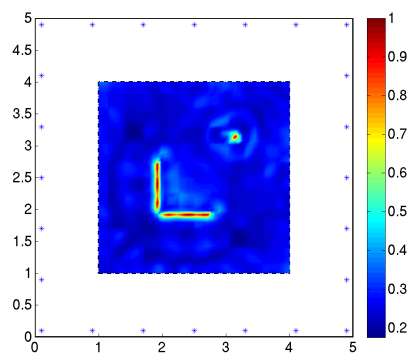

(c)

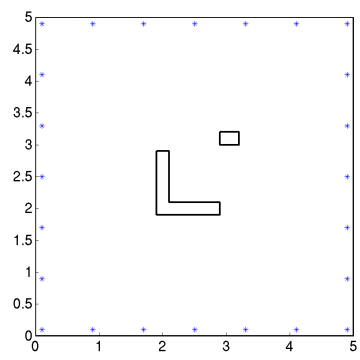

(f)

Figure 3: Reconstructions without artificial noise: (a) monopole test functions yielding the indicator $G^{(0)}$, see $(38)$ (b) dipole test functions yielding the indicator $G^{(1)}$, see $(38)$ (c) indicator $G^{(\max )}(z)$, see (39). Reconstruction with artificial noise: (d) indicator $G^{(\max )}(z)$, noise level $5 \%$ (e) indicator $G^{(\max )}(z)$, noise level $10 \%$ (f) geometry of the obstacles. The 24 emitters and receivers are marked by blue stars.

In the first numerical example we test the linear sampling algorithm against a non-convex obstacle consisting of two parts. The first component is an L-shaped obstacle with a Neumann boundary condition, the second component consists of a smaller obstacle where a Dirichlet boundary condition is imposed. We computed the scattered fields at the $N_{m}=N_{i}=24$ receivers between time $t=0$ and $t=28.9$, recording in total $N_{T}=413$ time steps. The discretized near-field operator $\mathrm{N}$ is hence represented by a square matrix of dimension $N_{T} N_{m}=9912$. For the reconstruction we computed 1650 singular values of this operator, which took 596 seconds. (The largest singular value is about 0.997 , the smallest one is about $0.429 \cdot 10^{-2}$.) Figure 3 indicates the reconstruction using the monopole test functions in (a), the reconstruction using dipoles in (b), and the maximum of both in (c). Clearly, the reconstruction using monopoles almost misses the Neumann obstacle (despite it is much bigger when compared to the Dirichlet obstacle). The dipole reconstruction shown in (b) is not very good in recovering the Dirichlet obstacle either. The combination of both indicators yields a good reconstruction shown in (c). The reconstructions in (d) and (e) are computed as in (c) but with noisy data by adding uniformly distributed random variables to the kernel of the discretized near-field operator N. The noise level has been set to 5 and $10 \%$ in (d) and 
(e), respectively, which still yields reconstructions providing relatively good geometric information, but smaller contrast.

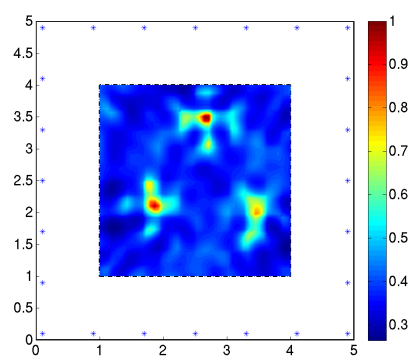

(a)

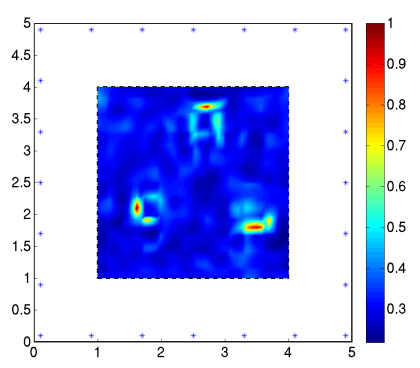

(b)

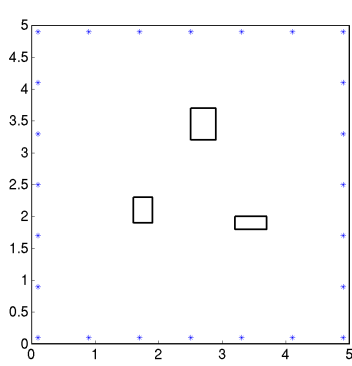

(c)

Figure 4: Reconstructions of Robin obstacles with 1\% artificial noise. (a) monopole test functions yielding the indicator $G^{(0)}$, see (38) (b) dipole test functions yielding the indicator $G^{(1)}$, see (38) (c) geometry of the obstacles

In the second numerical example we consider three obstacles with Robin boundary conditions. The geometry of the receivers is as in the first example and we record the scattered fields at 438 time steps between $t=0$ and $t=30.6$. The images in Figure 4 are computed using 2100 singular values and vectors of the discretized near-field operator of dimension 20512, the computation of the truncated singular system took 967 seconds. For the images in Figure 4 we used impedances $\alpha=0.15$ and $\alpha=0.2$ for the Robin boundary conditions. Numerical experiments showed that in this specific configuration such impedances yield Robin boundary conditions that are in an intermediate regime between the pure Dirichlet and Neumann conditions. Figure 4 shows that the dipole indicator function provides significantly more information on the obstacle shapes than the indicator function based on monopoles.

Figure 5 shows reconstructions using limited aperture data, using only six receivers on the lower side of the outer boundary. The geometry of the obstacles is as in the last example, see Figure 4(c), but the boundary conditions are chosen in a different way. On the lower left obstacle we prescribe a Dirichlet condition, while we prescribe an impedance boundary condition with $\alpha=0.2$ on the lower right and on the upper one. For the reconstruction, we used again 438 time steps, which yields a discrete near-field operator of dimension 3066. The reconstructions are based on 600 singular vectors and values; the computation time for the truncated singular value decomposition was 29 seconds. The reconstructions in Figure 5 all miss the distant, upper obstacle, but both identify the position of the lower obstacles correctly. The images computed using the monopole test functions tend to produce more concentrated reconstructions than those computed using dipole test functions.

Concluding, the numerical experiments show that the time domain linear sampling algorithm is relatively robust under noise and that it is to some extent possible to reconstruct obstacles from limited aperture data. Whenever one knows in advance that one faces an inverse scattering problem featuring obstacles with different physical properties, then we recommend not only to use the monopole test functions, but to try to extract information from the data using the dipole test functions, too. 


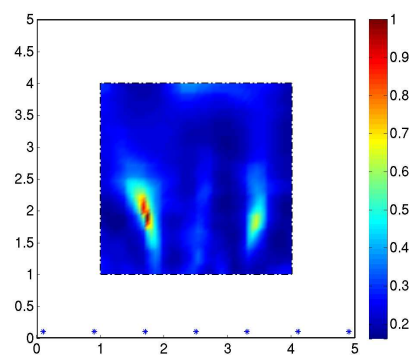

(a)

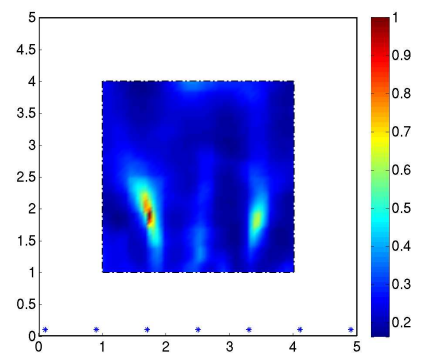

(d)

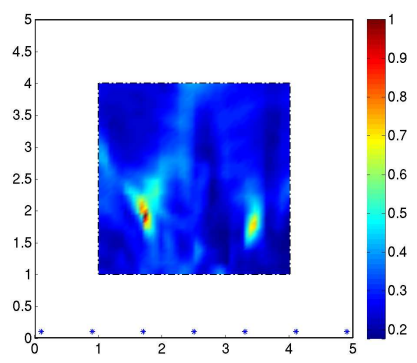

(b)

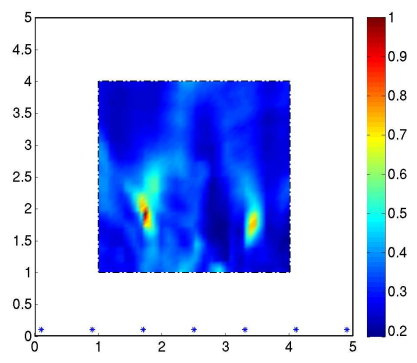

(e)

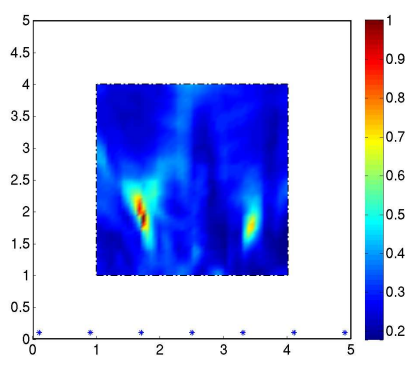

(c)

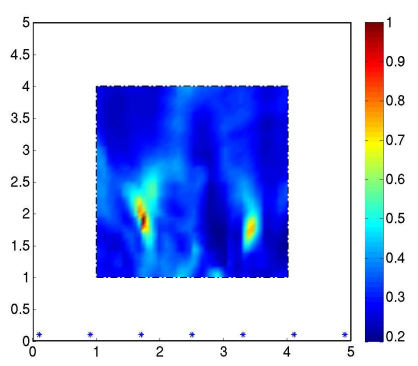

(f)

Figure 5: Reconstructions of Robin obstacles with 1\% artificial noise. (a) monopole test functions yielding the indicator $G^{(0)}$, see (38) (b) dipole test functions yielding the indicator $G^{(1)}$, see (38) (c) indicator $G^{(\max )}(z)$, see $(39)$. Reconstructions of Robin obstacles with $5 \%$ artificial noise. (d) monopole test functions yielding the indicator $G^{(0)}$, see $(38)$ (e) dipole test functions yielding the indicator $G^{(1)}$, see $(38)$ (f) indicator $G^{(\max )}(z)$, see $(39)$.

\section{A Some results on (retarded) potentials}

We summarize in this appendix some useful results from the literature on (retarded) potentials that has been used in the article. For details, we refer to [15, 9] or to [20. Let us consider an arbitrary Lipschitz surface $\Gamma \subset \mathbb{R}^{3}$ and formally introduce the single layer potential on $\Gamma$

$$
\left(L_{\Gamma} \psi\right)(t, x):=\int_{\Gamma} \frac{\psi(t-|x-y|, y)}{4 \pi|x-y|} d \sigma_{y},(t, x) \in \mathbb{R} \times\left(\mathbb{R}^{3} \backslash \Gamma\right) .
$$

Also of importance are the single and double layer operators on $\Gamma$

$$
\left(S_{\Gamma} \psi\right)(t, x):=\int_{\Gamma} \frac{\psi(t-|x-y|, y)}{4 \pi|x-y|} d \sigma_{y},(t, x) \in \mathbb{R} \times \Gamma
$$

and

$$
\left(K_{\Gamma} \psi\right)(t, x):=\int_{\Gamma} \partial_{n_{x}}\left(\frac{\psi(t-|x-y|, y)}{4 \pi|x-y|}\right) d \sigma_{y},(t, x) \in \mathbb{R} \times \Gamma .
$$

The operators $L_{\Gamma}, S_{\Gamma}$ and $K_{\Gamma}$ possess a convolution structure in the time variable and their Laplace transform are boundary integral operators. Indeed, for $\psi \in C_{0}^{\infty}(\mathbb{R} \times \Gamma)$ and $\omega \in\{z \in \mathbb{C}: \operatorname{Im} z>0\}$ 
one computes that

$$
\begin{array}{ll}
\widehat{L_{\Gamma} \psi}(\omega, x)=\frac{1}{4 \pi} \int_{\Gamma} \frac{e^{i \omega|x-y|}}{|x-y|} \widehat{\psi}(\omega, y) d \sigma_{y}=:\left(\widehat{L_{\Gamma}}(\omega) \widehat{\psi}(\omega, \cdot)\right)(x), & x \in \mathbb{R}^{3} \backslash \Gamma \\
\widehat{S_{\Gamma} \psi}(\omega, z)=\frac{1}{4 \pi} \int_{\Gamma} \frac{e^{i \omega|z-y|}}{|z-y|} \widehat{\psi}(\omega, y) d \sigma_{y}=:\left(\widehat{S_{\Gamma}}(\omega) \widehat{\psi}(\omega, \cdot)\right)(z), & z \in \Gamma \\
\widehat{K_{\Gamma} \psi}(\omega, z)=\frac{1}{4 \pi} \int_{\Gamma} \partial_{n_{z}}\left(\frac{e^{i \omega|z-y|}}{|z-y|} \widehat{\psi}(\omega, y)\right) d \sigma_{y}=:\left(\widehat{K_{\Gamma}}(\omega) \widehat{\psi}(\omega, \cdot)\right)(z), & z \in \Gamma .
\end{array}
$$

The following result is classical, see, e.g. [20]. For the rest of this section we suppose that $\Gamma$ is a closed Lipschitz surface, that the bounded connected component of $\mathbb{R}^{3} \backslash \Gamma$ is $\Omega_{-}$, and that $\Omega_{+}=\mathbb{R}^{3} \backslash \overline{\Omega_{-}}$.

Proposition 15. For $\omega \in\{z \in \mathbb{C}: \operatorname{Im} z>0\}$, the above potentials and boundary integral operators admit the following bounded extension

$$
\widehat{L_{\Gamma}}(\omega): H^{-1 / 2}(\Gamma) \rightarrow H^{1}\left(\mathbb{R}^{3}\right), \widehat{S_{\Gamma}}(\omega): H^{-1 / 2}(\Gamma) \rightarrow H^{1 / 2}(\Gamma), \widehat{K_{\Gamma}}(\omega): H^{-1 / 2}(\Gamma) \rightarrow H^{-1 / 2}(\Gamma) .
$$

Moreover, for $\psi \in H^{1 / 2}(\Gamma)$, we have the following jump relations

$$
\left(\widehat{L_{\Gamma}}(\omega) \psi\right)^{ \pm}=\widehat{S_{\Gamma}}(\omega) \psi, \quad \partial_{n}\left(\widehat{L_{\Gamma}}(\omega) \psi\right)^{\mp}=\left( \pm \frac{1}{2} I+\widehat{K_{\Gamma}}(\omega)\right) \psi
$$

where $(\cdot)^{-}$and $(\cdot)^{+}$denote the traces on $\Gamma$ taken from to $\Omega_{-}$and $\Omega_{+}$, respectively.

The following result that can be found, e.g., in [15], gives bounds on the frequency-dependence of these operators.

Proposition 16. Let $\sigma>0$. There exists a constant $C>0$ depending only on $\Gamma$ and $\sigma$ such that for all $\psi \in H^{-1 / 2}(\Gamma)$ and all $\omega \in \mathbb{R}+i \sigma$ it holds that

$$
\left\|\widehat{L_{\Gamma}}(\omega) \psi\right\|_{1, \omega, \Omega_{+}}+\left\|\widehat{S_{\Gamma}}(\omega) \psi\right\|_{1 / 2, \omega, \Gamma}+\left\|\widehat{K_{\Gamma}}(\omega) \psi\right\|_{-1 / 2, \omega, \Gamma} \leq C|\omega|\|\psi\|_{-1 / 2, \omega, \Gamma}
$$

Moreover, for all all $\omega \in \mathbb{R}+i \sigma$, the operator $\widehat{S_{\Gamma}}(\omega): H^{-1 / 2}(\Gamma) \rightarrow H^{1 / 2}(\Gamma)$ is invertible and there exists a constant $C>0$ depending only on $\Gamma$ and $\sigma$ such that for all $\varphi \in H^{1 / 2}(\Gamma)$ it holds that

$$
\left\|\widehat{S_{\Gamma}}(\omega)^{-1} \varphi\right\|_{-1 / 2, \omega, \Gamma} \leq C|\omega|\|\varphi\|_{1 / 2, \omega, \Gamma} .
$$

Let $\Sigma$ be a surface embedded into $\Omega_{+}$which is either a part of a closed analytic surface $\tilde{\Sigma}$ surrounding $\Omega_{+}$or the boundary of a Lipschitz bounded domain containing $\Omega_{+}$. In the first case, the space $H^{1 / 2}(\Sigma)$ is defined as the restriction to $\Sigma$ of functions in $H^{1 / 2}(\tilde{\Sigma})$. The following result can be seen as a consequence of the proof of [7, Lemma 9].

Proposition 17. Let $\sigma>0$. Then the operator $\operatorname{tr}_{\Sigma} \widehat{L_{\Gamma}}(\omega): H^{-1 / 2}(\Gamma) \rightarrow H^{1 / 2}(\Sigma)$ is injective with dense range.

Let $X$ be a Hilbert space, we define for $p \in \mathbb{R}$ and $\sigma \in \mathbb{R}$,

$$
H_{\sigma}^{p}(\mathbb{R}, X):=\left\{g \in \mathcal{L}_{\sigma}^{\prime}(\mathbb{R}, X) \text { such that } \int_{-\infty+i \sigma}^{\infty+i \sigma}|\omega|^{2 p}\|\hat{g}(\omega)\|_{X}^{2} d \omega<+\infty\right\}
$$

The next lemma is a simple consequence of the Plancherel identity for Fourier-Laplace multipliers in a vector-valued setting (compare, e.g. [18]). 
Lemma 18. Let $X$ and $Y$ be two Hilbert spaces and assume that

$$
\mathcal{A}: \mathcal{D}(\mathbb{R}, X) \rightarrow \mathcal{D}^{\prime}(\mathbb{R}, Y), \quad g \mapsto \int_{\mathbb{R}} A(t-\tau) g(\tau) d \tau
$$

is a time convolution operator with kernel $A \in \mathcal{L}_{\sigma}^{\prime}(\mathbb{R}, \mathcal{L}(X, Y))$ for some $\sigma \in \mathbb{R}$. We assume that the Laplace transform of $A$ denoted by $\omega \in \mathbb{R}+i \sigma \mapsto \widehat{A}(\omega) \in \mathcal{L}(X, Y)$ is locally integrable and satisfies

$$
\|\widehat{A}(\omega)\|_{\mathcal{L}(X, Y)} \leq C|\omega|^{s} \text { for a.e. } \omega \in \mathbb{R}+i \sigma
$$

and for some $s \in \mathbb{R}$. Then $A$ admits a bounded extension to a linear operator from $H_{\sigma}^{p+s}(\mathbb{R}, X)$ into $H_{\sigma}^{p}(\mathbb{R}, Y)$, for all $p \in \mathbb{R}$.

Combining the the previous estimate with Lemma[18 yields the following bounds for the retarded potentials and operators in the time domain.

Proposition 19. Let $p \in \mathbb{R}$ and $\sigma>0$. The operators

$$
L_{\Gamma}: H_{\sigma, \Gamma}^{p,-1 / 2} \rightarrow H_{\sigma, \Omega_{+}}^{p-1,1}, \quad S_{\Gamma}: H_{\sigma, \Gamma}^{p,-1 / 2} \rightarrow H_{\sigma, \Gamma}^{p-1,1 / 2}, \quad \text { and } \quad K_{\Gamma}: H_{\sigma, \Gamma}^{p,-1 / 2} \rightarrow H_{\sigma, \Gamma}^{p-1,-1 / 2}
$$

are bounded. Moreover, for $\psi \in H_{\sigma, \Gamma}^{p,-1 / 2}$ the following jump relations hold,

$$
\left(L_{\Gamma} \psi\right)^{ \pm}=S_{\Gamma} \psi \quad \text { in } H_{\sigma, \Gamma}^{p-1,1 / 2} \quad \text { and } \quad \partial_{n}\left(L_{\Gamma} \psi\right)^{\mp}=\left( \pm \frac{1}{2} I+K_{\Gamma}\right) \psi \quad \text { in } H_{\sigma, \Gamma}^{p-1,-1 / 2}
$$

\section{References}

[1] H. Ammari, An introduction to mathematics of emerging biomedical imaging, vol. 62 of Mathematics \& Applications, 2008.

[2] A. Bamberger And T. H. Duong, Formulation variationnelle espace-temps pour le calcul par potentiel retardé de la diffraction d'une onde acoustique, Mathematical Methods in the Applied Science, 8 (1986), pp. 405-435.

[3] K. Bingham, Y. Kurylev, M. Lassas, and S. Siltanen, Iterative time-reversal control for inverse problems, Inverse Problems and Imaging, 2 (2008), pp. 63-81.

[4] L. Borcea, G. C. Papanicolaou, C. Tsogka, and J. Berryman, Imaging and time reversal in random media, Inverse Problems, 18 (2002), pp. 1247-1279.

[5] C. Burkard and R. Potthast, A time-domain probe method for three-dimensional rough surface reconstructions, Inverse Problems and Imaging, 3 (2009), pp. 259-274.

[6] F. Cakoni and D. Colton, Qualitative Methods in Inverse Scattering Theory. An Introduction., Springer, Berlin, 2006.

[7] Q. Chen, H. Haddar, A. Lechleiter, and P. Monk, A sampling method for inverse scattering in the time domain, Inverse Problems, 26 (2010).

[8] D. Colton And R. Kress, Inverse Acoustic and Electromagnetic Scattering Theory, Springer, 1992. 
[9] M. Costabel, Time-dependent problems with the boundary integral equation method, Encyclopedia of Computational Mechanics, (2004).

[10] R. Dautray and J. Lions, Analyse mathématiques et calcul numérique pour les sciences et techniques, Masson, 1985.

[11] M. Filipe, A. Forestier, And T. HA-Duong, A time-dependent acoustic scattering problem, in Third International Conference on Mathematical and Numerical Aspects of Wave Propagation, G. C. Cohen, ed., SIAM, 1995, pp. 140-150.

[12] M. Frigo And S. G. Johnson, The design and implementation of FFTW3, Proceedings of the IEEE, 93 (2005), pp. 216-231.

[13] R. GRIESMAIER, Multi-frequency orthogonality sampling for inverse obstacle scattering problems, Inverse Problems, 27 (2011), p. 085005.

[14] B. Guzina, F. Cakoni, And C. Bellis, On the multi-frequency obstacle reconstruction via the linear sampling method, Inverse Problems, 26 (2010), p. 125005 (29pp).

[15] T. HA-Duong, On retarded potential boundary integral equations and their discretisations, in Topics in Computational Wave Propagation: Direct and Inverse Problems, M. Ainsworth, P. Davies, D. Duncan, P. Martin, and B. Rynne, eds., Springer-Verlag, 2003, pp. 301-336.

[16] H. Haddar, A. Lechleiter, and S. Marmorat, Une méthode d'échantillonnage linéaire dans le domaine temporel : le cas des obstacles de type Robin-Fourier, Research Report RR7835, INRIA, Nov. 2011.

[17] A. Kirsch and N. Grinberg, The Factorization Method for Inverse Problems, Oxford Lecture Series in Mathematics and Its Applications, 2008.

[18] C. Lubich, On the multistep time discretization of linear initial-boundary value problems and their boundary integral equations, Numerische Mathematik, 67 (1994), pp. 365-389.

[19] D. R. Luke And R. Potthast, The point source method for inverse scattering in the time domain, Math. Meth. Appl. Sci., 29 (2006), pp. 1501-1521.

[20] W. McLean, Strongly Elliptic Systems and Boundary Integral Equations, Cambridge University Press, 2000.

[21] L. OKSANEN, Inverse obstacle problem for the non-stationary wave equation with an unknown background, Preprint, http://arxiv.org/abs/1106.3204, (2011).

[22] N. T. Thánh AND M. Sini, Accuracy of the linear sampling method for inverse obstacle scattering: effect of geometrical and physical parameters, Inverse Problems, 26 (2010), p. 125004.

[23] F. Treves, Basic Linear Partial Differential Equations, Academic Press, 1975. 